\title{
Sorted gene genealogies and species-specific nonsynonymous substitutions point to putative postmating prezygotic isolation genes in Allonemobius crickets
}

Suegene Noh, Daniel J Howard, Jeremy L Marshall

In the Allonemobius socius complex of crickets, reproductive isolation is primarily accomplished via postmating prezygotic barriers. We tested seven protein-coding genes expressed in the male ejaculate for patterns of evolution consistent with a putative role as postmating prezygotic isolation genes. Because our species generally lacked sequence variation in these genes, we found approaches that took advantage of evolutionary processes specific to young species were more successful at identifying putative postmating prezygotic isolation genes: lineage sorting in gene trees and allele sharing in haplotype networks. Only some of our genes showed evidence of elevated $\omega$ values on the internal branches of gene trees, and in a couple genes these internal branches coincided with both species branching events of the species tree, between $A$. fasciatus and the other two species and between $A$. socius and $A$. sp. nov. Tex. These approaches were particularly powerful within the contact zone. Among the genes we tested we found few genes with genealogies that indicated relatively advanced degrees of lineage sorting across both allopatric and contact zone alleles. Within a contact zone between two members of the species complex, only a subset of genes maintained allelic segregation despite evidence of ongoing gene flow in other genes. The overlap in all these analyses were arginine kinase (AK) and apolipoprotein A-1 binding protein (APBP), two of the first examples of sperm maturation, capacitation, and motility proteins with fixed nonsynonymous substitutions between species-specific alleles that may lead to postmating prezygotic isolation. Both genes express ejaculate proteins transferred to females during copulation and were previously identified through comparative proteomics. We discuss the potential function of these genes in the context of the specific postmating prezygotic isolation phenotype among our species, namely conspecific sperm precedence and the superior ability of conspecific males to induce females to lay eggs. 
1 Sorted gene genealogies and species-specific non-synonymous substitutions point to

2 putative postmating prezygotic isolation genes in Allonemobius crickets

3

4 Suegene Noh $^{*}, 1$, Daniel J. Howard ${ }^{2,3}$, Jeremy L. Marshall ${ }^{4}$

$5 \quad{ }^{1}$ Department of Biology, Washington University in St. Louis, St. Louis, Missouri 63130, USA

62 Department of Integrative Biology, University of Colorado Denver, Denver, Colorado 80217, 7 USA

$8{ }^{3}$ Office of the Executive Vice President and Provost, New Mexico State University, Las Cruces,

9 New Mexico 88003, USA

$10{ }^{4}$ Department of Entomology, Kansas State University, Manhattan, Kansas 66506, USA

11 * Corresponding author: Suegene Noh, Department of Biology, Washington University in St.

12 Louis, One Brookings Drive, Campus Box 1137, St. Louis, Missouri 63130, USA

13 E-mail: suegene.noh@gmail.com

15 Running title: Postmating prezygotic isolation genes

16 Word count: 6811

17 Data Archival Location: Sequences formatted as haplotypes are available from NCBI GenBank

18 PopSets 372477571 (ACG69), 372477483 (AK), 372477513 (APBP), 372477527 (EJAC-SP),

19372477535 (GOT), 372477555 (SPAG6), 372477561 (SPI).. 


\section{Abstract}

22 In the Allonemobius socius complex of crickets, reproductive isolation is primarily accomplished

23 via postmating prezygotic barriers. We tested seven protein-coding genes expressed in the male

24 ejaculate for patterns of evolution consistent with a putative role as postmating prezygotic

25 isolation genes. Because our species generally lacked sequence variation in these genes, we

26 found approaches that took advantage of evolutionary processes specific to young species were

27 more successful at identifying putative postmating prezygotic isolation genes: lineage sorting in

28 gene trees and allele sharing in haplotype networks. Only some of our genes showed evidence of

29 elevated $\omega$ values on the internal branches of gene trees, and in a couple genes these internal

30 branches coincided with both species branching events of the species tree, between $A$. fasciatus

31 and the other two species and between $A$. socius and $A$. sp. nov. Tex. These approaches were

32 particularly powerful within the contact zone. Among the genes we tested we found few genes

33 with genealogies that indicated relatively advanced degrees of lineage sorting across both

34 allopatric and contact zone alleles. Within a contact zone between two members of the species

35 complex, only a subset of genes maintained allelic segregation despite evidence of ongoing gene

36 flow in other genes. The overlap in all these analyses were arginine kinase (AK) and

37 apolipoprotein A-1 binding protein (APBP), two of the first examples of sperm maturation,

38 capacitation, and motility proteins with fixed non-synonymous substitutions between species-

39 specific alleles that may lead to postmating prezygotic isolation. Both genes express ejaculate

40 proteins transferred to females during copulation and were previously identified through

41 comparative proteomics. We discuss the potential function of these genes in the context of the

42 specific postmating prezygotic isolation phenotype among our species, namely conspecific

43 sperm precedence and the superior ability of conspecific males to induce females to lay eggs. 


\section{Introduction}

45 Not all genes contribute equally to reproductive isolation during speciation. 'Speciation' (Wu, 46 2001; Wu \& Ting, 2004; Nosil \& Schluter, 2011), 'isolation' (Rieseberg, Church \& Morjan, 47 2004), or 'barrier' (Noor \& Feder, 2006) genes are expected to show very different patterns of evolution compared to genes that are not directly involved in reproductive isolation when species are still undergoing lineage sorting (Wu, 2001; Feder, Egan \& Nosil, 2012). Therefore we expect to find putative speciation genes among those genes that become fixed for alternative alleles within each incipient species early in the process of divergence, with said alleles rarely crossing the species boundary in sympatry (Ting, Tsaur \& Wu, 2000; Dopman et al., 2005). important role in the evolution of postmating prezygotic reproductive isolation. Many reproductive genes are known to evolve rapidly in a variety of organisms (Civetta \& Singh, 1998; Swanson \& Vacquier, 2002; Clark, Aagaard \& Swanson, 2006; Panhuis \& Swanson, 2006; Snook et al., 2009). In Drosophila where some of the most extensive work has been done, genes that show male-biased expression evolve faster compared to female-biased and somatically expressed genes (Zhang, Hambuch \& Parsch, 2004; Zhang \& Parsch, 2005; Metta et al., 2006; Pröschel, Zhang \& Parsch, 2006; Haerty et al., 2007), and seminal fluid proteins in particular tend to show an excess of non-synonymous substitutions (Begun et al., 2000; Swanson et al., 2001; Wagstaff \& Begun, 2005; Almeida \& DeSalle, 2008). Similar patterns have also been observed in mice and primates (Clark \& Swanson, 2005; Karn et al., 2008; Ramm et al., 2008; Turner, Chuong \& Hoekstra, 2008; Dean et al., 2009). Using a proteomics approach on insect spermatophores to isolate male reproductive protein coding-genes that can directly interact with 
67 putative speciation genes (Andrés, Maroja \& Harrison, 2008; Marshall et al., 2011; Andrés et al., 68 2013).

69 The male ejaculate proteome comprises sperm-expressed proteins and seminal fluid

70 proteins. Sperm not only contribute half of the diploid genome, but are also involved in sperm-

71 egg interactions including egg activation and deliver paternal factors during fertilization (Dorus

72 et al., 2006). Seminal fluid proteins, the majority of which are produced by male accessory

73 glands, contain conserved functional classes of peptides and pro-hormones that are involved in

74 sperm binding, proteolysis, lipid metabolism, and immune function (Mueller et al., 2004;

75 Chapman \& Davies, 2004; Poiani, 2006; Avila et al., 2011). Once transferred into the female reproductive tract, these proteins can initiate a wide-range of physiological functions including increased egg production and oviposition, decreased receptivity, decreased lifespan, and increased feeding in females (reviewed in (Avila et al., 2011). The interacting female counterparts to these ejaculate proteins (EPs) are not as well known (Ram, Ji \& Wolfner, 2005; Ram \& Wolfner, 2007; Snook et al., 2009) though genomic data is proving to be invaluable for identifying candidates (Baer et al., 2009a; Findlay et al., 2014). The evolution of EPs has been hypothesized to be driven by one or more processes including female sperm preference, sperm competition, and sexual conflict (Mueller et al., 2004; Snook et al., 2009).

The A. socius complex of ground crickets, A. socius, A. fasciatus, and A. sp. nov. Tex, represents a powerful system to explore the hypothesized link between EP divergence and reproductive isolation. Members of this complex are primarily isolated from one another by two

87 postmating, prezygotic phenotypes - conspecific sperm precedence (Gregory \& Howard, 1994;

88 Howard et al., 1998a,b; Marshall, 2004) and the superior ability of conspecific males to induce

89 females to lay eggs (Gregory \& Howard, 1993; Howard et al., 1998b). Two other compelling 
90 features of this organismal system are species boundaries that remain intact in sympatry despite

91 some gene flow (Howard, 1986; Howard \& Waring, 1991; Traylor et al., 2008) and the very

92 recent nature of divergence between these species (i.e., within the last 30,000 years; (Marshall,

93 2004, 2007). Indeed, divergence is so recent that few species-specific alleles have been identified;

94 for example, only 2 of 17 allozyme markers (Howard, 1983), 2 of 5,400 AFLP markers (Howard

95 et al., 2002), 21 of 1,660 thorax proteins and $\sim 33$ of 922 ejaculate proteins (Marshall et al.,

96 2011) and 1 of 16 randomly chosen reproductive genes spanning $>7,500$ bp of coding sequence

97 (Marshall et al., unpublished data), yield evidence of species specificity. Taken together, the

98 above data suggest that while there is sufficient genetic divergence to produce reproductive

99 isolation and maintain species boundaries in sympatry, the vast majority of genes show little

100 evidence of divergence. In all, the $A$. socius complex represents a system whereby speciation is

101 ongoing with potentially relatively few genes contributing to the postmating prezygotic

102 reproductive isolation between species. Therefore, if we can identify those ejaculate and female

103 reproductive tract genes that exhibit signatures of positive selection and maintain species-

104 specificity in sympatry, we will gain insight into the postmating prezygotic isolation genes that

105 are ultimately driving speciation in this system.

106 In this study, we examine EPs in the A. socius complex to identify genes that show

107 patterns of sequence evolution and lineage sorting that are consistent with a potential

108 contribution to postmating prezygotic isolation between species. We examined seven EP coding

109 genes, five of which were identified in a comparative proteome study between the $A$. socius and

110 A. fasciatus (Marshall et al., 2011) and two additional EP coding genes identified from

111 unpublished EST libraries of $A$. socius accessory glands and testes (Marshall et al., 2011). The

112 five EP coding genes from the comparative proteome study were previously tested for evidence 
113 of positive selection with limited population sampling and sequence fragments. When testing for

114 evidence of selection in recently diverged species biases can arise when a supposed fixation is in

115 fact a polymorphism, when a true fixation is an ancestral polymorphism rather than a new

116 mutation, and because neutral and adaptive mutations fix at different rates (Keightley \& Eyre-

117 Walker, 2012). We expanded population sampling across the species ranges for the two species

118 that were tested previously, added the third species $A$. sp. nov. Tex and examined longer

119 sequence lengths to test whether our previous conclusions hold up to expanded sampling, at both

120 the population and species level and at the sequence level. Finally, we observed how sequences

121 of these genes behave within and across the contact zone of $A$. fasciatus and $A$. socius by looking

122 for evidence of allele sharing and lineage sorting. These combined analyses point toward an

123 important role for some but not all examined EPs during the evolution of reproductive isolation

124 within this complex of crickets.

125

126 Methods

127 Background

128 Striped ground crickets of the A. socius complex inhabit moist grasslands across North

129 America and do not show significant habitat isolation (Howard 1986). The three species $A$.

130 socius, $A$. fasciatus, and $A$. sp. nov. Tex form two contact zones, one between $A$. fasciatus (north)

131 and $A$. socius (south) from Illinois to New Jersey (Howard \& Waring, 1991), and one between $A$.

132 sp. nov. Tex (west) and A. socius (east) near the Louisiana - Texas state line (Traylor et al.,

133 2008). A. fasciatus and $A$. socius seem to have diverged from a common ancestor approximately

13430,000 years ago, and $A$. sp. nov. Tex seems to have subsequently diverged from $A$. socius

135 approximately 24,000 years ago (Marshall, 2004, 2007). They have previously been shown to be 
136 isolated primarily via postmating prezygotic reproductive isolation (Howard et al., 2002;

137 Marshall, 2004; Marshall \& DiRienzo, 2012).

139 Population and gene sampling

140 Crickets were collected from each population in the summer of 2010 and genotyped in

141 the lab via allozymes (Isocytrate dehydrogenase and Hexokinase) to determine species identity

142 (Howard, 1983; Marshall 2004; Traylor et al 2008). Sampling localities spanned the range of

143 each species. The seven $A$. socius populations were sampled near Texarkana, AR (AR), Bottom,

144 NC (Bot), Mt. Vernon, IL (IL), Pleasantville, NJ (Mi), Ruston, LA (LA), Gastonia, NC (NC),

145 and Ardmore, $\mathrm{OK}(\mathrm{OK})$. The three A. fasciatus populations were sampled near Akron, $\mathrm{OH}$ (Akn),

146 Frankfort, IL (FF), and New Paltz, NY (NP). The three A. sp. nov. Tex populations were sampled

147 near Terrell, TX (Tx20), Royse City, TX (Tx30), and Gainesville, TX (Tx35). Contact zone

148 populations of $A$. fasciatus and $A$. socius were sampled from two habitats at a single location in

149 Kenna, WV. A. fasciatus was collected from a hillside habitat, which we call Kenna Hill (KH),

150 and A. socius was collected along the base of hill near a creek which we call Kenna Creek (KC).

151 We did not have samples from the contact zone between $A$. socius and A. sp. nov. Tex. General

152 maintenance protocols followed Marshall et al (2009). Briefly, juveniles were reared to maturity

153 in population and sex-specific plastic cages. All crickets were maintained at $27 \mathrm{C}$ and $14: 10 \mathrm{~h}$

154 light to dark photoperiod.

155 We dissected male accessory glands and testes from three individuals per allopatric 156 population and 9 individuals per contact zone population. cDNA was synthesized from each

157 tissue using RNA isolated via an Ambion RNAqueous-4PCR (\#AM1914) kit and standard

158 protocols for $1^{\text {st }}$ strand cDNA synthesis. General PCR and sequencing procedures followed 
159 Marshall et al (2011). Our reagent amounts for a $25 \mu \mathrm{L}$ reactions were: $2.510 x$ buffer B, 2.0

$160 \mathrm{MgCl}_{2}(25 \mathrm{mM}), 0.5 \mathrm{dNTP}(10 \mathrm{mM}), 0.5$ for each primer $(10 \mu \mathrm{M}), 1 \mathrm{U}$ Taq (Fisher), $0.5 \mathrm{cDNA}$,

$16118.3 \mathrm{ddH}_{2} \mathrm{O}$. We used the following PCR program: $94{ }^{\circ} \mathrm{C}$ for $2: 00 \mathrm{~min}$, then 30 cycles of $94{ }^{\circ} \mathrm{C}$

162 for $30 \mathrm{sec}$, annealing at $45-55^{\circ} \mathrm{C}$ for $30 \mathrm{sec}, 72{ }^{\circ} \mathrm{C}$ for $1: 00 \mathrm{~min}$, and final elongation at $72{ }^{\circ} \mathrm{C}$

163 for 7:00 min. Specific annealing temperatures depended on individual primer melting

164 temperatures (primers used are shown in Supplementary Table 1). Sequencing was done at the

165 Kansas State University Department of Plant Pathology DNA Sequencing and Genotyping

166 Facility using Applied Biosystems Big Dye chemistry on an Applied Biosystems 3730 DNA

167 Analyzer.

168 We compared nucleotide sequences of seven EP coding genes: 1) acg69 (ACG69), a

169 novel protein of unknown function expressed in the accessory glands; 2) arginine kinase (AK), a

170 phosphagen kinase that catalyzes ATP-regeneration and energy transport in invertebrates and

171 some protozoa (Ellington, 2001; Noguchi, Sawada \& Akazawa, 2001; Uda et al., 2006); 3)

172 apolipoprotein A-1 binding protein (APBP), a phosphoprotein expressed in sperm that is

173 homologous to a mammalian sperm capacitation gene (Jha et al., 2008); 4) ejaculate serine

174 protease (EJAC-SP), an abundant accessory gland-expressed serine protease previously shown

175 to be involved in the induction of egg laying in successfully mated females (Marshall et al.,

176 2009); 5) serine protease inhibitor (SPI), a testis-expressed serine-type endopeptidase inhibitor;

177 6) aspartate aminotransferase (GOT), a pyridoxal-phospate-dependent aminotransferase

178 expressed in the testis that is also an allozyme historically used to diagnose species identity

179 among A. socius complex crickets (Howard, 1983, 1986); 7) sperm-associated antigen 6

180 (SPAG6), homologous to a mammalian protein important for sperm flagellar motility and the

181 structural integrity of the central apparatus (Neilson et al., 1999; Sapiro et al., 2002). The first 
182 five genes were investigated to a lesser extent in a comparison of male ejaculate proteome

183 profiles in $A$. fasciatus and $A$. socius (Marshall et al., 2011). On 2D-DIGE (differential in-gel

184 electrophoresis) gels ACG69, EJAC-SP and SPI had non species-specific protein spots that

185 indicate similar molecular weights, isoelectric points, and expression levels in the male ejaculate,

186 while AK and APBP had species-specific spots that indicate differences in one or more of these

187 proteomic traits. The latter two genes were EP coding genes present in EST libraries of $A$. socius

188 accessory glands and testes (Marshall et al., 2011) that were identified as potential candidates

189 based on a review of sperm biology literature.

190 Sequences formatted as haplotypes are available from NCBI GenBank PopSets

191372477571 (ACG69), 372477483 (AK), 372477513 (APBP), 372477527 (EJAC-SP),

192372477535 (GOT), 372477555 (SPAG6), 372477561 (SPI),.

193

194 Species tree-based analyses

195 We first applied tests of selection to the species tree of the $A$. socius complex (A.

196 fasciatus, (A. socius, A. sp. nov. Tex)). The ratio of non-synonymous to synonymous substitution

197 rates $\omega$ is widely used to detect signatures of selection acting upon protein coding genes (Yang \&

198 Bielawski, 2000; Nielsen, 2001, 2005; Jensen, Wong \& Aquadro, 2007). When $\omega$ is larger than 1,

199 positive or balancing selection is inferred. When $\omega$ is smaller than 1 , negative or purifying

200 selection is inferred. We aligned all sequences in BioEdit v.7.0.5.3 (Hall, 1999). We counted

201 within species polymorphisms and between species fixations and calculated $\pi_{\mathrm{s}}, \pi_{\mathrm{a}}$ and $\theta=4 \mathrm{~N}_{\mathrm{e}} \mu$

202 across all populations with DnaSP v.5.10.1 (Librado \& Rozas, 2009). Unless stated otherwise,

203 the majority of the remaining analyses were carried out in HyPhy v.2.2.1 (Kosakovsky Pond,

204 Frost \& Muse, 2005) or its online server Datamonkey (Delport et al., 2010). We selected a 
205 nucleotide substitution model (NucModelCompare.bf) at a model rejection level of 0.0002 , the

206 recommended level based on Bonferroni correction of comparing 203 increasingly parameter-

207 rich nucleotide substitution models (Kosakovsky Pond \& Frost, 2005a). Next we tested for

208 evidence of recombination using the Genetic Algorithm Recombination Detection (GARD)

209 method (SingleBreakpointRecomb.bf). Recombination should be ruled out or accounted for

210 because its presence can mislead inferences of selection due to no single phylogenetic tree being

211 able to accurately describe the evolutionary relationship of recombinant sequences (Anisimova,

212 Nielsen \& Yang, Ziheng, 2003; Shriner et al., 2003; Kosakovsky Pond et al., 2006). GARD tests

213 for evidence of recombination by comparing fits of a single tree vs segment-specific trees to

214 alignments. Segments are potential recombinant fragments, as defined by sequence in between

215 possible breakpoints (variable sites).

216 Next we fit maximum likelihood models to the species tree to estimate $\omega$ at each

217 branching node: the node between A. fasciatus and the other two species, and the node between

218 A. socius and A. sp. nov. Tex. Using the codon substitution model MG94 (Muse \& Gaut, 1994)

219 while estimating codon frequencies based on combinations of nucleotide frequencies (called $3 \times 4$

220 in HyPhy), we fit and optimized a maximum likelihood model on the multiple sequence

221 alignment for each gene, and jointly estimated both $\omega$ and their fine asymptotic normal CI

222 estimates from the same likelihood model. HyPhy derives CI estimates analytically from the

223 Fisher Information Matrices of the log likelihood surface of each model parameter (Kosakovsky

224 Pond \& Muse, S. V., 2005).

225 Finally, we compared polymorphism within species to divergence between species at

226 each branching node of the species tree. We compared $\omega$, the rate ratios of synonymous

227 substitutions per synonymous site $\kappa_{\mathrm{a}}\left(d_{\mathrm{N}}\right)$ and non-synonymous substitutions per non- 
228 synonymous site $\kappa_{\mathrm{s}}\left(d_{\mathrm{S}}\right)$ for each gene using McDonald-Kreitman tests (McDonald, Kreitman \&

229 others, 1991) in DnaSP, and across all genes using a multilocus HKA test (Hudson, Kreitman \&

230 Aguadé, 1987) with the program HKA (Wang \& Hey, 1996). The null model of the McDonald-

231 Kreitman test is that the ratio of non-synonymous to synonymous intraspecific polymorphisms

232 should be no different from the ratio of non-synymous to synonymous fixations between species.

233 The multilocus HKA test uses coalescent simulations to test the null model that all loci within a

234 species will share the same effective population size, and loci between sister species will evolve

235 under the same neutral mutation rate. The HKA program also reports an outlier (Wang \& Hey,

236 1996) that is the observation that deviates the most from its expected value, corresponding to a

237 specific locus and its level of polymorphism within species or fixation between species.

238

239 Gene tree-based analyses

240 Gene trees show the evolutionary histories of individual genes and are less likely to

241 concur with species trees the shorter the time since divergence due to incomplete lineage sorting

242 and introgression (Pamilo \& Nei, 1988; Maddison, 1997). Because genes more directly related to

243 reproductive isolation and speciation should show patterns of evolution more closely resembling

244 the species tree (Wu, 2001; Feder, Egan \& Nosil, 2012), comparing gene trees should inform us

245 of, or confirm, which genes are more likely to be the key genes involved in postmating

246 prezygotic isolation, particularly when evidence suggests the action of selection upon internal

247 nodes that separate incipient species.

248 Using Neighbor-Joining gene trees built using Tamura-Nei distances (Tamura \& Nei,

249 1993) in HyPhy, we tested for evidence of selection on specific internal branches of the gene tree

250 that had non-zero branch length (TestBranchdNdS.bf). This method estimates the non- 
251 synonymous rate while assuming a single synonymous substitution rate across a tree, so in effect

252 tests for evidence of elevated $\omega$ on specific branches. We used the MG94 and F81 (Felsenstein,

253 1981) substitution models and fit a single non-synonymous substitution rate across the tree with

254 no within branch variation in $\kappa_{\mathrm{s}}$ or $\kappa_{\mathrm{a}}$. We compared this model to a model where the internal

255 nodes of interest (those whose non-synonymous substitution rate's 95\% CI did not overlap with

256 zero based on a first pass run of this method on all internal branches with non-zero branch

257 lengths) were allowed to vary from the global non-synonymous rate using likelihood ratio tests.

258 We used MEGA6 (Tamura et al., 2013) to visualize these trees.

259 We tested for evidence of specific sites evolving under different selection regimes using

260 the single likelihood ancestor counting (SLAC) method (Kosakovsky Pond \& Frost, 2005b).

261 SLAC (QuickSelectionDetection.bf) tests all sites of each gene for evidence of selection by first

262 taking a tree and fitting a codon model (MG94 x F81) to obtain a global estimate of $\omega=\kappa_{\mathrm{s}} / \kappa_{\mathrm{a}}$. It

263 then reconstructs an ancestral sequence for each site across all internal nodes of the tree using

264 joint maximum likelihood estimation, taking into account the estimate of $\omega$ obtained in the

265 previous step. Then for each variable site, the method compares expected and observed numbers

266 of synonymous and non-synonymous substitutions to detect selection.

Finally, we tested for evidence of episodic selection anywhere within each gene using a

268 branch-site method. BUSTED (branch-site unrestricted statistical test for episodic diversification)

269 tests whether there is evidence suggesting any single gene is under positive selection, while

270 accounting for site-level variation in selection and variable selection on a subset of branches on a

271 phylogenetic tree (Murrell et al., 2015). We partitioned branches into two categories: those of

272 interest (foreground), which were the same branches from the branch method above

273 (TestBranchdNdS) that had non-zero branch lengths, and the remainder (background). BUSTED 
274 optimizes the likelihood using a random effects likelihood framework (Kosakovsky Pond et al.,

275 2011) and first fits an unconstrained model that proportions sites in the foreground branches into

276 one of three variable rates including those over $\omega=1$. This alternative model is then compared to

277 a null model in which all rates are constrained to $\omega=<1$, but all sites are still fit and

278 proportioned into three rates. These models are compared to each other using likelihood ratio

279 tests.

280

281 Evidence from the contact zone between $\mathrm{A}$. fasciatus and A. socius

282 The genealogical sorting index (gsi) reflects the degree of lineage sorting of individual gene

283 genealogies that occurs during speciation, with values ranging from zero (complete polyphyly) to

2841 (complete monophyly) (Cummings, Neel \& Shaw, 2008). We calculated gsi for each gene

285 using the gsi web service (www.molecularevolution.org) with gene trees including both

286 allopatric and contact zone individuals. We generated gene trees for gsi analysis with Neighbor

287 Joining in TreeBeST v.1.9.2 (Li, 2006) using the option ntmm that calculates p-distances from

288 codon alignments. We used this method because gsi requires a rooted tree and TreeBeST can use

289 a species tree to root a gene tree using an algorithm (Zmasek \& Eddy, 2011) that compares

290 potential root positions on an unrooted tree and places the root where the differences between the

291 gene tree and species tree are minimized $(\mathrm{Li}, 2006)$. The program permutes the labels (in our

292 case, species identity) of the tips of the given tree multiple times (we used the default $n=10000$ ),

293 each time determining the gsi value of this new tree. The permuted P-value is the probability of

294 randomly observing a gsi value equal to or better (higher) than the gsi value observed from the

295 data. We used MEGA6 to visualize these trees. 
Finally, we constructed statistical parsimony haplotype networks (Templeton, Crandall \&

297 Sing, 1992) of alleles from all three species to test for species-specificity of alleles. We used

298 TCS v.1.21 (Clement, Posada \& Crandall, 2000) to generate the haplotype networks using only

299 allopatric individuals. Species-specific alleles were defined as those found only within each

300 respective species. Common or shared alleles were those observed in more than one species.

301 Once alleles were designated common or specific to a species, we looked at nine individuals

302 each within the contact zone of $A$. fasciatus and $A$. socius and determined what types of allele

303 these contact zone individuals possessed. As noted above, these individuals had previously been

304 designated as fully (homozygous) A. fasciatus or $A$. socius based on allozymes. To compare the

305 allelic distributions of these genes, we calculated a segregation metric, the dissimiliarity index $D$

306 (Duncan \& Duncan, 1955) using the R package SEG (Hong, O’Sullivan \& Sadahiro, 2014).

307 Duncan and Duncan's $D$ is a measure of segregation in space that ranges from 0 (complete

308 integration) to 1 (complete segregation). For genes for which data was missing (1 individual for

309 the gene SPAG6), we calculated the potential range of $D$ depending on the potential values for

310 the missing observations.

311

312 Results

313 Species tree-based analyses

314 We fit the nucleotide substitution model F81 to all genes, and found no evidence of

315 recombination in any of our genes at any of the variable sites. We found relatively low levels of

316 both synonymous and non-synonymous nucleotide variation within and among the $A$. socius

317 complex species. The Watterson estimator $\theta$ ranged from 0.001 to $0.011, \kappa_{\mathrm{s}}$ ranged from 0.004 to

3180.054 , and $\kappa_{\mathrm{a}}$ ranged from 0 to 0.009 (Table $1 \& 2$ ). Next we estimated $\omega=\kappa_{\mathrm{a}} / \kappa_{\mathrm{s}}$ at each 
319 branching event of the species tree. In the older split between $A$. fasciatus and the other two

320 species the maximum likelihood estimates of $\omega$ exceeded 1 , indicating evidence of positive or

321 balancing selection in the genes AK $(\omega=24.841,95 \%$ CI $12.988 \sim 36.694)$, APBP $(\omega=13.495$,

$32295 \%$ CI $0 \sim 35.670)$, and SPI $(\omega=1.356,95 \%$ CI $0 \sim$ Inf $)$, while EJAC-SP approached $\omega=1(\omega$

$323=0.993,95 \%$ CI $0 \sim 16.673$ ). In the younger split between $A$. socius and A. sp. nov. Tex $\omega$ did

324 not exceed 1 in any of the genes (Table 2). Because of the combination of low $\pi_{\mathrm{s}}, \pi_{\mathrm{a}}$, and $\theta$ and

325 high $\omega$ for these genes, we will henceforth interpret $\omega>1$ as evidence of positive selection as

326 balancing selection is more likely to be accompanied by a higher nucleotide diversity.

$327 \quad$ Not all genes had fixed non-synonymous substitutions between species and in these cases

328 we were unable to apply the McDonald-Kreitman test (Table 2). For those genes that were

329 testable, we did not find significant differences in $D_{\mathrm{N}} / D_{\mathrm{S}}$ compared to $P_{\mathrm{N}} / P_{\mathrm{S}}$ at either branching

330 event (Fisher's exact test $P=0.07 \sim 1$ ). We were unable to detect a significant departure from

331 neutral expectations for the first branching event between $A$. fasciatus and the two other species

332 using the multilocus HKA test $\left(X^{2} P=0.916\right)$. We did detect a significant departure from neutral

333 expectations for the second branching event between $A$. socius and $A$. sp. nov. Tex. $\left(X^{2} P=\right.$

334 0.012). The outlier that diverged most from null expectations was polymorphism in GOT in $A . s p$.

335 nov. Tex., but coalescent simulations were unable to determine that this was a significant

336 difference $(P=0.06)$. Both the McDonald-Kreitman and HKA tests are likely to be

337 underpowered for our species given the age and amount of variation present in the Drosophila

338 systems that both tests were originally developed for (Hudson, Kreitman \& Aguadé, 1987;

339 McDonald, Kreitman \& others, 1991; Wang \& Hey, 1996).

340

341 Gene tree-based analyses 
We tested for evidence that any of the internal branches of each gene tree were evolving

343 at a higher non-synonymous rate than the other branches (Figure 1). We found evidence that the

344 model in which two internal nodes in the gene tree of AK were allowed to evolve at a variable

345 non-synonymous rate was a better fit to the single rate class model $(P=0.004)$. These nodes

346 evolved at non-synonymous substitution rates $26.331(4.377-81.378)$ and $0.147(0.008-0.649)$,

347 while the shared non-synonymous substitution rate was estimated to be 0 . The first node (5) is

348 the node that separates $A$. fasciatus and the two other species on the AK gene tree. The second

349 node (43) is the node that separates $A$. socius and $A$. sp. nov. Tex on the AK gene tree. We also

350 found evidence that two internal nodes in the gene tree of APBP were evolving at a different rate

351 compared to the background branches $(P=0.015)$. These nodes were evolving at rates 43.793

352 (2.515 - 194.273) and 19.008 (1.088 - 84.035), while the shared non-synonymous substitution

353 rate was estimated to be 0 . The first node (11) is the node between A. fasciatus and the two other

354 species on the APBP gene tree. The second node (45) is the node between A. socius and A. sp.

355 nov. Tex on the APBP gene tree. Despite evidence that some internal nodes were evolving at a

356 higher rate compared to background nodes in the other genes, we did not have sufficient

357 evidence to suggest branches in any of the other gene trees were evolving at more than one non-

358 synonymous rate class (ACG69 (6 tested) $P=0.171$, EJAC-SP ( 1 tested) $P=0.277$, GOT (3

359 tested) $P=0.218$, SPI ( 1 tested) $P=0.265$, SPAG6 (2 tested) $P=1$ ).

360 Using SLAC and comparing model fits of nucleotide substitution models and codon

361 models, for most of genes (ACG69, AK, APBP, SPI, SPAG6) we found no evidence of any

362 specific sites evolving under positive or negative selection. For EJAC-SP, one site (position 115)

363 in which the amino acid serine was maintained through synonymous substitutions, showed

364 evidence of negative selection $(P=0.011)$. Also for GOT, one site (position 88 ) in which the 
365 animo acid threonine was maintained through synonymous substitutions, showed evidence of

366 negative selection $(P=0.037)$.

367 We used the method BUSTED in HyPhy to test for episodic selection anywhere in each

368 gene. We tested whether any sites evolved at a faster $\omega$ rate within the internal branches with

369 non-zero branches tested in the previous branch analysis. We found non-significant evidence of

370 episodic selection only in ACG69 using this method (Likelihood Ratio Test $P=0.062$ ). The

371 unconstrained model suggests $1.7 \%$ of foreground branch sites may be evolving with $\omega=318.77$

372 while background branch sites have a maximum $\omega=0.96(100 \%$ of the sites were partitioned

373 into this rate class). This branch-site test is likely to be underpowered for our species given that

374 the numbers of parameters that were estimated for the unconstrained model was upwards of 80

375 (depending on the number of terminal taxa for each gene), while the difference in numbers of

376 parameters between the complex model and null model was only 1 . We do not discuss results

377 from this method further because results were non-significant across all genes.

378

379 Evidence from the contact zone between $\mathrm{A}$. fasciatus and $\mathrm{A}$. socius

380 Comparisons of genealogical sorting index values based on gene trees including all

381 sampled individuals, both allopatric and contact zone, indicated that lineage sorting was ongoing

382 as gsi values ranged from 0 to 1 , with a median value of 0.836 . If we use the median value as a

383 cutoff, only AK and APBP showed relatively advanced lineage sorting for all three species while

384 GOT showed advanced lineage sorting for A. fasciatus and A. sp. nov. Tex but not A. socius

385 (Table 3, Figure 2).

386 The statistical parsimony haplotype networks generated using allopatric individuals of all

387 three species showed that alleles in AK, APBP, and GOT were specific to each species, while the 
388 alleles present in other genes included both species-specific alleles and alleles shared between

389 two species (Figure 3). Within the contact zone between A. fasciatus and A. socius, all genes

390 except APBP had a mix of alleles that we had already observed in allopatric individuals as well

391 as new alleles that were specific to the contact zone (Figure 2). We designated these new alleles

392 as species-specific based on their allelic distributions among contact zone individuals and

393 calculated the degree of allelic segregation within the contact zone. The dissimilarity index $D$

394 values we observed were bimodally distributed and indicated allelic segregation was higher in

395 the contact zone for AK, APBP, EJACSP and SPAG6 $(D=0.771 \sim 1)$ and lower in ACG69,

396 GOT and SPI $(D=0.164 \sim 0.353)$ (Figure 4$)$.

397

398 Discussion

Studying speciation in recently diverged species is attractive because we can potentially

400 identify variation that is associated with divergence and not accumulated after (Andrés et al.,

401 2013). But using within and between species patterns of sequence variation to detect adaptive

402 evolution is particularly challenging in recently diverged species because less evolutionary time

403 has passed to allow for fixed differences to accumulate between incipient species relative to

404 within species polymorphism (Keightley \& Eyre-Walker, 2012). We gathered data from

405 populations across each species distribution so as to accurately differentiate between

406 polymorphisms and fixations and detect new mutations to confirm and expand upon previous

407 results from more limited population sampling (Marshall et al., 2011). Combining evidence from

408 multiple tests allowed us to identify the most likely putative postmating prezygotic isolation

409 genes among our EP coding genes (Table 4). Ultimately, the methodological approaches that we 
410 were able to apply most successfully were those that took advantage of evolutionary processes

411 specific to young species: lineage sorting in gene trees and allele sharing in haplotype networks.

412 Data from the relatively recently ( $\sim 30,000$ years) diverged $A$. socius species complex

413 showed a general lack of both synonymous and non-synonymous nucleotide variation within and

414 among all investigated genes (Table $1 \& 2$ ). Our estimates of sequence variation were also at

415 least an order of magnitude smaller compared to other known estimates from accessory gland

416 protein coding genes in various other species groups (Mueller et al., 2005; Wagstaff \& Begun,

417 2005; Almeida \& DeSalle, 2008), including some Gryllus crickets whose species are of roughly

418 similar age (Andrés et al., 2006). Since speciation in the A. socius complex is thought to coincide

419 with glaciation history (Marshall, 2004, 2007) population bottlenecks may have contributed to

420 sequence variation patterns. Our results would have benefited from increased within-population

421 sampling so that metrics such as Tajima's or Fu and Li's $D$ and site frequency spectra, as well as

422 estimates of population size and recombination rates (Nielsen, 2005) could be accurately applied.

423 Unsurprisingly, the tests we used that relied on sequence variation on the known species tree had

424 minor success identifying genes with evidence of positive selection based on $\omega$ (Table 4). The

425 maximum likelihood estimates of $\omega$ on the species tree indicated that AK, APBP, EJAC-SP and

426 SPI showed evidence of positive selection between the branching event between $A$. fasciatus and

427 the other two species (Table 2). However McDonald-Kreitman tests were not applicable to

428 several genes and HKA tests were generally inconclusive.

429 We found approaches that used gene trees and haplotype networks were more successful

430 at detecting evidence of positive selection, and these approaches were particularly powerful

431 within the contact zone (Table 4). When allopatric gene trees were tested for evidence of internal

432 branches evolving at different rates compared to the rest of the tree, we found evidence that AK 
433 and APBP both had internal branches were evolving at higher non-synonymous substitution rates

434 (Figure 1). These internal branches respectively separated A. fasciatus from the other two species, 435 and A. socius from A. sp. nov. Tex. When all allopatric and contact zone individuals were

436 examined, the genealogies of AK and APBP indicated that these genes were relatively advanced

437 in their degrees of lineage sorting in all three species of the $A$. socius complex compared to the

438 other genes (Table 3, Figure 2). GOT also showed advanced lineage sorting, but only in the two

439 species A. fasciatus and A. sp. nov. Tex. Within the contact zone of A. fasciatus and A. socius,

440 AK, APBP, EJAC-SP, and SPAG6 showed highly segregated allelic distributions (Figure 4). The

441 two genes with intermediate lineage sorting or allelic segregation patterns, EJAC-SP and GOT,

442 showed evidence of purifying selection acting upon specific sites, as might be expected of most

443 functional protein coding genes (Lawrie et al., 2013), and no evidence of positive selection. The

444 overlap among these results suggests AK and APBP are the most likely candidates for

445 postmating prezygotic isolation genes (Table 4). While the remaining genes that show

446 intermediate patterns may still contribute to reproductive isolation, they are less likely to be the

447 major contributors to isolation barriers that act in a species-specific manner.

448 These patterns fit models of ongoing speciation in the face of gene flow, where

449 incomplete lineage sorting and introgression are major confounding factors (Machado \& Hey,

450 2003; Broughton \& Harrison, 2003; Payseur, 2010). Speciation genes are more likely to become

451 fixed for species-specific alleles early in the process of speciation and therefore are expected to

452 be relatively exempt from incomplete sorting and subject to reduced introgression (Wu, 2001;

453 Feder, Egan \& Nosil, 2012). Similar patterns have been observed in Drosophila, field crickets,

454 and moths (Ting, Tsaur \& Wu, 2000; Dopman et al., 2005; Maroja, Andrés \& Harrison, 2009;

455 Andrés et al., 2013; Larson et al., 2013). It is possible that these genes are not the direct targets 
456 but rather linked to targets of divergent selection as the interaction between linkage and selection

457 makes it challenging to distinguish between recurrent positive selection, background selection, 458 and Hill-Robertson effects (Hill \& Robertson, 1966; Charlesworth, 1994; Andolfatto, 2007;

459 Charlesworth et al., 2009). Because both genes were identified as candidates through 460 comparative proteomics (Marshall et al., 2011) this seems relatively unlikely, but the genomic

461 regions around these genes should be investigated for evidence of selective sweeps to rule out 462 this possibility.

463 Many studies of reproductive proteins report evidence of positive selection acting on a 464 subset of the genes examined, in both males (Begun et al., 2000; Swanson et al., 2001; Clark \& 465 Swanson, 2005; Wagstaff \& Begun, 2005; Andrés et al., 2006; Karn et al., 2008; Ramm et al., 466 2008; Almeida \& DeSalle, 2008; Walters \& Harrison, 2010) and females (Swanson et al., 2004;

467 Panhuis \& Swanson, 2006; Lawniczak \& Begun, 2007; Prokupek et al., 2008; Kelleher \& 468 Markow, 2009; Kelleher, Clark \& Markow, 2011). However, there are few examples of adaptive 469 reproductive protein evolution leading to reproductive isolation outside of gamete recognition 470 proteins (e.g. (Geyer \& Palumbi, 2003; McCartney \& Lessios, 2004; Springer \& Crespi, 2007).

471 Our putative postmating prezygotic isolation genes AK and APBP are two of the first examples 472 of sperm maturation and capacitation related proteins that show evidence of fixed non473 synonymous substitutions between species-specific alleles leading to reproductive isolation 474 (Figure 3). We had previously observed this pattern between A. fasciatus and A. socius for both 475 AK and APBP (Marshall et al., 2011), but finding the same pattern in the mutational steps 476 between $A$. socius and $A$. sp. nov. Tex with different species-specific non-synonymous 477 substitutions, with expanded population and sequence sampling, emphasizes the potential 478 importance of these candidates. 
Whether there are functional consequences to the species-specific non-synonymous

480 substitutions in AK and APBP needs to be investigated further. Both candidates are homologous

481 to mammalian sperm capacitation proteins. Sperm maturation and capacitation occur inside the

482 female reproductive tract of mammals (Visconti et al., 2011). In insects and nematodes, sperm

483 are capacitated and become motile by serine proteases present in the seminal fluid (LaFlamme \&

484 Wolfner, 2013). This process occurs within the spermatophores of Lepidoptera (Osanai \& Chen,

485 1993; Friedländer, Jeshtadi \& Reynolds, 2001), and in the seminal vesicles of Drosophila who

486 do not make spermatophores (Osanai \& Chen, 1993). In either case, most insect sperm should

487 then be already mature and capacitated once spermatophores are transferred to females.

488 Therefore the specific hypothetical function of these genes should be related to a different

489 postcopulatory process that occurs within the female reproductive tract.

$490 \quad$ Female insects store sperm in specialized organs such as spermathecae and seminal

491 receptacles after copulation, often for prolonged periods of time (Schnakenberg, Siegal \& Bloch

492 Qazi, 2012). Sperm lose motility within a day in flies, unless stored by females into these organs

493 (Schnakenberg, Matias \& Siegal, 2011). Conspecific sperm precedence among Drosophila

494 species has been shown to involve post-copulatory processes that occur within the female

495 reproductive tract that include sperm transfer, the displacement and ejection of less preferred

496 (heterospecific) sperm, and the selective use of preferred (conspecific) sperm from different

497 storage organs for fertilization (Manier et al., 2013a,b). In flies, spermathecal secretory cells are

498 intimately involved in the female driven part of these processes (Schnakenberg et al 2011).

499 Endopeptidases produced by these secretory cells are necessary for recruiting sperm to

500 spermathecae and also maintaining sperm motility in the seminal receptacle. The same

501 endopeptidases also affect egg laying, so that females laid fewer eggs when their secretory cells 
502 were ablated. More recently, it was shown that the number of secretory cells that produce these

503 endopeptidases determined whether female flies ovulated and layed eggs (Sun \& Spradling,

504 2013). It is yet unknown whether sequence variation in these endopeptidases is related to

505 variation in female fecundity. Other potential roles for the secretions of these cells as related to

506 sperm precedence include a chemotactic function that would attract select sperm to different

507 locations of the female reproductive tract (Wolfner, 2011).

508 The specific mechanism of conspecific sperm precedence that APBP is involved in may

509 be related to the appropriate phosphorylation state of the phosphoprotein (APBP) depending on

510 the female vs. male species combination. APBP becomes phosphorylated during murine sperm

511 capacitation and co-localizes with cholesterol during this process, but its specific function is

512 unknown (Jha et al., 2008). As noted above, insect sperm do not undergo capacitation as

513 mammalian sperm do. As a putative binding protein with an enzymatic function, hypothetical

514 functions of APBP in the female reproductive tract include that as a signal for sperm to be

515 transferred to the preferred storage organ. It may also be involved in a signaling cascade that

516 induces ovulation and oviposition in females. Between A. fasciatus and A. socius, when females

517 were mated with only a single male those mated with heterospecific males laid fewer eggs but an

518 equal proportion of fertilized eggs compared to females mated with conspecific males (Gregory

519 \& Howard, 1993).

520 Insects and other ecdysozoans possess AK as their sole phosphagen system for cellular

521 energy metabolism, and accordingly, arginine phosphate and its phosphagen kinase AK are

522 found primarily in muscles, but also in sperm and compound eyes (Strong \& Ellington, 1993;

523 Kucharski \& Maleszka, 1998; Ellington, 2001). The possible roles of AK as an EP can be related

524 to sperm motility or the acrosome reaction (Strong \& Ellington, 1993; Niksirat et al., 2015). As 
525 an energy-related phosphagen kinase, AK may confer to sperm an enhanced ability to move 526 toward a sperm storage organ and resist displacement and ejection by females. In honeybees, AK

527 is expressed in both male seminal fluid (Baer et al., 2009b) and female spermathecal fluid (Baer

528 et al., 2009a). When tested over a two-year period AK enzymatic activity decreased in mated

529 female spermathecal fluid and sperm motility also decreased (Al-Lawati, Kamp \& Bienefeld, 530 2009). The proteomes of freshly ejaculated vs. stored honeybee sperm are also known to change, 531 particularly in terms of reduced activity of glycolytic enzymes that should be used for energy

532 metabolism (Poland et al., 2011). Poland and colleagues note that most of the same enzymes are

533 also present in the spermathecal fluid (Baer et al., 2009a), suggesting female physiology has an

534 active role in maintaining stored sperm. Two structural loops and several active sites near them

535 are the proposed interaction interface of AK with the guanidinium groups of its substrates (Zhou

536 et al., 1998; Pruett et al., 2003; Azzi et al., 2004; Clark, Davulcu \& Chapman, 2012). As might

537 be expected for an integral enzyme, the non-synonymous substitutions we observed do not occur 538 at these specific sites, though they may still influence its activity.

\section{Conclusions}

541 A. socius complex crickets provide an excellent opportunity to identify patterns of

542 evolution in speciation genes for two major reasons: speciation is incomplete as evidenced by

543 ongoing gene flow in the field, and isolation is through a single type of reproductive isolation

544 barrier, i.e., postmating prezygotic phenotypes. We found that when speciation is ongoing, 545 combining multiple approaches, particularly those that incorporate evidence from gene trees and

546 haplotype networks were most effective at identifying putative postmating prezygotic isolation

547 genes. Both AK and APBP have fixed, or nearly fixed, non-synonymous substitutions between 
548 both A. fasciatus and the other two species, and between A. socius and A. sp. nov. Tex. Both

549 genes showed advanced lineage sorting across and within the contact zone, and allelic

550 segregation within the contact zone. The next step is to determine the functional consequence of

551 allelic variation in either EP in conspecific sperm precedence or the successful induction of

552 female oviposition in the A. socius complex.

553

554 Acknowledgments

555 We thank Christopher Garcia for collecting the crickets used in this study and Diana

556 Huestis and Shanda Wheeler for their help in isolating RNA and screening individuals for

557 species status with allozymes. This is contribution no. 12-015-J from the Kansas Agricultural

558 Experiment Station. 


\section{References}

561 Almeida FC, DeSalle R. 2008. Evidence of adaptive evolution of accessory gland proteins in

562 closely related species of the Drosophila repleta group. Molecular Biology and Evolution

$563 \quad 25: 2043-2053$.

564 Andolfatto P. 2007. Hitchhiking effects of recurrent beneficial amino acid substitutions in the Drosophila melanogaster genome. Genome Research 17:1755-1762.

566

567

568

569

570

571

572

573

574

575

576

577

578

579

580

581

Andrés JA, Maroja LS, Bogdanowicz SM, Swanson WJ, Harrison RG. 2006. Molecular evolution of seminal proteins in field crickets. Molecular Biology and Evolution $23: 1574-1584$.

Andrés JA, Larson EL, Bogdanowicz SM, Harrison RG. 2013. Patterns of transcriptome divergence in the male accessory gland of two closely related species of field crickets. Genetics 193:501-513.

Andrés JA, Maroja LS, Harrison RG. 2008. Searching for candidate speciation genes using a proteomic approach: seminal proteins in field crickets. Proceedings of the Royal Society of London B: Biological Sciences 275:1975-1983.

Anisimova M, Nielsen R, Yang, Ziheng. 2003. Effect of recombination on the accuracy of the likelihood method for detecting positive selection at amino acid sites. Genetics $164: 1229-1236$.

Avila FW, Sirot LK, LaFlamme BA, Rubinstein CD, Wolfner MF. 2011. Insect seminal fluid proteins: identification and function. Annual review of entomology 56:21-40.

Azzi A, Clark SA, Ellington WR, Chapman MS. 2004. The role of phosphagen specificity loops in arginine kinase. Protein Science 13:575-585. 
582 Baer B, Eubel H, Taylor NL, O’Toole N, Harvey Millar A. 2009a. Insights into female sperm

583

584

585

586

587 storage from the spermathecal fluid proteome of the honeybee Apis mellifera. Genome Biology 10:R67.

Baer B, Heazlewood JL, Taylor NL, Eubel H, Harvey Millar A. 2009b. The seminal fluid proteome of the honeybee Apis mellifera. Proteomics 9:2085-2097.

Begun DJ, Whitley P, Todd BL, Waldrip-Dail HM, Clark AG. 2000. Molecular population genetics of male accessory gland proteins in Drosophila. Genetics 156:1879-1888.

Broughton RE, Harrison RG. 2003. Nuclear gene genealogies reveal historical, demographic and selective factors associated with speciation in field crickets. Genetics 163:1389-1401.

Chapman T, Davies SJ. 2004. Functions and analysis of the seminal fluid proteins of male Drosophila melanogaster fruit flies. Peptides 25:1477-1490.

Charlesworth B. 1994. The effect of background selection against deleterious mutations on weakly selected, linked variants. Genetical Research 63:213-227.

Charlesworth B, Betancourt AJ, Kaiser VB, Gordo I. 2009. Genetic recombination and molecular evolution. Cold Spring Harbor Symposium on Quantitative Biology 74:177186.

Civetta A, Singh RS. 1998. Sex and speciation: genetic architecture and evolutionary potential of sexual versus nonsexual traits in the sibling species of the Drosophila melanogaster complex. Evolution:1080-1092.

Clark NL, Aagaard JE, Swanson WJ. 2006. Evolution of reproductive proteins from animals and plants. Reproduction 131:11-22. 
603 Clark SA, Davulcu O, Chapman MS. 2012. Crystal structures of arginine kinase in complex with 604 ADP, nitrate, and various phosphagen analogs. Biochemical and Biophysical Research $605 \quad$ Communications 427:212-217.

606 Clark NL, Swanson WJ. 2005. Pervasive adaptive evolution in primate seminal proteins. PLoS $607 \quad$ Genetics 1:e35.

608 Clement M, Posada D, Crandall KA. 2000. TCS: a computer program to estimate gene 609 genealogies. Molecular ecology 9:1657-1659.

610 Cummings MP, Neel MC, Shaw KL. 2008. A genealogical approach to quantifying lineage 611 divergence. Evolution 62:2411-2422.

612 Dean MD, Clark NL, Findlay GD, Karn RC, Yi X, Swanson WJ, MacCoss MJ, Nachman MW. 613 2009. Proteomics and comparative genomic investigations reveal heterogeneity in 614 evolutionary rate of male reproductive proteins in mice (Mus domesticus). Molecular 615 Biology and Evolution 26:1733-1743.

616 Delport W, Poon AF, Frost SD, Pond SLK. 2010. Datamonkey 2010: a suite of phylogenetic analysis tools for evolutionary biology. Bioinformatics 26:2455-2457.

618 Dopman EB, Pérez L, Bogdanowicz SM, Harrison RG. 2005. Consequences of reproductive barriers for genealogical discordance in the European corn borer. Proceedings of the National Academy of Sciences of the United States of America 102:14706-14711.

621 Dorus S, Busby SA, Gerike U, Shabanowitz J, Hunt DF, Karr TL. 2006. Genomic and functional 622 623 evolution of the Drosophila melanogaster sperm proteome. Nature genetics 38:14401445.

624 Duncan OD, Duncan B. 1955. A methodological analysis of segregation indexes. American $625 \quad$ Sociological Review 20:210-217. 
626 Ellington WR. 2001. Evolution and the physiological roles of phosphagen systems. Annual

627 Review of Physiology 63:289-325.

628 Feder JL, Egan SP, Nosil P. 2012. The genomics of speciation-with-gene-flow. Trends in $629 \quad$ Genetics 28:342-350.

630 Felsenstein J. 1981. Evolutionary trees from DNA-sequences: a maximum-likelihood approach. 631 Journal of Molecular Evolution 17:368-376.

632 Findlay GD, Sitnik JL, Wang W, Aquadro CF, Clark NL, Wolfner MF. 2014. Evolutionary rate 633 covariation identifies new members of a protein network required for Drosophila melanogaster female post-mating responses. PLoS Genetics 10:e1004108.

635 Friedländer M, Jeshtadi A, Reynolds S. 2001. The structural mechanism of trypsin-induced 636 637 intrinsic motility in Manduca sexta spermatozoa in vitro. Journal of Insect Physiology $47: 245-255$.

Geyer LB, Palumbi SR. 2003. Reproductive character displacement and the genetics of gamete recognition in tropical sea urchins. Evolution 57:1049-1060.

640 Gregory PJ, Howard DJ. 1993. Laboratory hybridization studies of Allonemobius fasciatus and $A$. 641 socius (Orthoptera: Gryllidae). Annals of the Entomological Society of America 86:694642 701.

Gregory PJ, Howard DJ. 1994. A post-insemination barrier to fertilization isolates two closely 644 related ground crickets. Evolution 48:705-710.

645 Haerty W, Jagadeeshan S, Kulathinal RJ, Wong A, Ram KR, Sirot LK, Levesque L, Artieri CG, 646 Wolfner MF, Civetta A, others. 2007. Evolution in the fast lane: rapidly evolving sex647 related genes in Drosophila. Genetics 177:1321-1335. 
648 Hall TA. 1999. BioEdit: a user-friendly biological sequence alignment editor and analysis 649 program for Windows 95/98/NT. In: Nucleic Acids Symposium Series. 95-98.

650 Hill WG, Robertson A. 1966. The effect of linkage on limits to artificial selection. Genetical 651 Research 8:269-294.

652 Hong S-Y, O’Sullivan D, Sadahiro Y. 2014. Implementing spatial segregation reasures in R. PLOS ONE 9:e113767.

Howard DJ. 1983. Electrophoretic survey of eastern North American Allonemobius (Orthoptera: Gryllidae): evolutionary relationships and the discovery of three new species. Annals of the Entomological Society of America 76:1014-1021.

Howard DJ. 1986. A zone of overlap and hybridization between two ground cricket species. Evolution:34-43.

Howard DJ, Gregory PJ, Chu J, Cain ML. 1998a. Conspecific sperm precedence is an effective barrier to hybridization between closely related species. Evolution 52:511-516.

661 Howard DJ, Reece PG, Gregory PJ, Chu J, Cain ML. 1998b. The evolution of barriers to fertilization between closely related organisms. In: Howard DJ, Berlocher SH eds. Endless Forms: Species and Speciation. New York, NY: Oxford University Press, 279_

Howard DJ, Marshall JL, Hampton DD, Britch SC, Draney ML, Chu J, Cantrell RG. 2002. The 666 genetics of reproductive isolation: a retrospective and prospective look with comments on ground crickets. American Naturalist 159:S8-S21.

668 Howard DJ, Waring GL. 1991. Topographic diversity, zone width, and the strength of 669 reproductive isolation in a zone of overlap and hybridization. Evolution:1120-1135. 
670 Hudson RR, Kreitman M, Aguadé M. 1987. A test of neutral molecular evolution based on 671 nucleotide data. Genetics 116:153-159.

672 Jensen JD, Wong A, Aquadro CF. 2007. Approaches for identifying targets of positive selection. $673 \quad$ Trends in Genetics 23:568-577.

674 Jha KN, Shumilin IA, Digilio LC, Chertihin O, Zheng H, Schmitz G, Visconti PE, Flickinger CJ, 675 Minor W, Herr JC. 2008. Biochemical and structural characterization of apolipoprotein 676 AI binding protein, a novel phosphoprotein with a potential role in sperm capacitation. Endocrinology 149:2108-2120.

678

679 680 681 682 683 684 685 686 687 688 689

Karn RC, Clark NL, Nguyen ED, Swanson WJ. 2008. Adaptive evolution in rodent seminal vesicle secretion proteins. Molecular Biology and Evolution 25:2301-2310.

Keightley PD, Eyre-Walker A. 2012. Estimating the rate of adaptive molecular evolution when the evolutionary divergence between species is small. Journal of Molecular Evolution $74: 61-68$.

Kelleher ES, Clark NL, Markow TA. 2011. Diversity-enhancing selection acts on a female reproductive protease family in four subspecies of Drosophila mojavensis. Genetics $187: 865-876$.

Kelleher ES, Markow TA. 2009. Duplication, selection and gene conversion in a Drosophila mojavensis female reproductive protein family. Genetics 181:1451-1465.

Kosakovsky Pond SL, Posada D, Gravenor MB, Woelk CH, Frost SDW. 2006. GARD: a genetic algorithm for recombination detection. Bioinformatics 22:3096-3098.

Kosakovsky Pond SLK, Murrell B, Fourment M, Frost SDW, Delport W, Scheffler K. 2011. A random effects branch-site model for detecting episodic diversifying selection. Molecular Biology and Evolution 28:3033-3043. 
693 Kosakovsky Pond SL, Frost SDW. 2005a. A simple hierarchical approach to modeling

694 distributions of substitution rates. Molecular Biology and Evolution 22:223-234.

695 Kosakovsky Pond SL, Frost SDW. 2005b. Not so different after all: a comparison of methods for 696 detecting amino-acid sites under selection. Molecular Biology and Evolution 22:1208$697 \quad 1222$.

698 Kosakovsky Pond SL, Frost SDW, Muse SV. 2005. HyPhy: hypothesis testing using phylogenies. 699 Bioinformatics 21:676-679.

700 Kosakovsky Pond SL, Muse, S. V. 2005. HyPhy: hypothesis testing using phylogenies. In:

701 Nielsen R ed. Statistical Methods for Molecular Evolution. Statistics for Biology and 702 Health. New York, NY: Springer, 125-181.

703 Kucharski R, Maleszka R. 1998. Arginine kinase is highly expressed in the compound eye of the 704 honey-bee, Apis mellifera. Gene 211:343-349.

705 LaFlamme BA, Wolfner MF. 2013. Identification and function of proteolysis regulators in 706 seminal fluid. Molecular Reproductive Development 80:80-101.

707 Larson EL, Andrés JA, Bogdanowicz SM, Harrison RG. 2013. Differential introgression in a 708 mosaic hybrid zone reveals candidate barrier genes. Evolution; International Journal of $709 \quad$ Organic Evolution 67:3653-3661.

710 Al-Lawati H, Kamp G, Bienefeld K. 2009. Characteristics of the spermathecal contents of old 711 and young honeybee queens. Journal of Insect Physiology 55:116-121.

712 Lawniczak MK, Begun DJ. 2007. Molecular population genetics of female-expressed mating713 induced serine proteases in Drosophila melanogaster. Molecular Biology and Evolution 714 24:1944-1951. 
715 Lawrie DS, Messer PW, Hershberg R, Petrov DA. 2013. Strong purifying selection at

716 synonymous sites in D. melanogaster. PLoS Genet 9:e1003527.

717 Li H. 2006. Constructing the TreeFam database. Doctoral Dissertation Thesis. The Institute of

718 Theoretical Physics, Chinese Academy of Science.

719 Librado P, Rozas J. 2009. DnaSP v5: a software for comprehensive analysis of DNA

$720 \quad$ polymorphism data. Bioinformatics 25:1451-1452.

721 Machado CA, Hey J. 2003. The causes of phylogenetic conflict in a classic Drosophila species

722 group. Proceedings of the Royal Society of London B: Biological Sciences 270:1193-

723 1202.

724 Maddison WP. 1997. Gene trees in species trees. Systematic Biology 46:523-536.

725 Manier MK, Lüpold S, Belote JM, Starmer WT, Berben KS, Ala-Honkola O, Collins WF, 726 Pitnick, S. 2013a. Postcopulatory sexual selection generates speciation phenotypes in Drosophila. Current Biology 23:1853-1862.

728

729

730

731

732

733

734

735

736

737

Manier MK, Belote JM, Lüpold S, Berben KS, Ala-Honkola O, Collins WF, Pitnick S. 2013 b. Rapid diversification of sperm precedence traits and processes among three sibling Drosophila species. Evolution 67:2348-2362.

Maroja LS, Andrés JA, Harrison RG. 2009. Genealogical discordance and patterns of introgression and selection across a cricket hybrid zone. Evolution 63:2999-3015.

Marshall JL. 2004. The Allonemobius-Wolbachia host-endosymbiont system: evidence for rapid speciation and against reproductive isolation driven by cytoplasmic incompatibility. Evolution 58:2409-2425.

Marshall JL. 2007. Rapid evolution of spermathecal duct length in the Allonemobius socius complex of crickets: species, population and Wolbachia effects. PLoS One 2:e720. 
738 Marshall JL, Huestis DL, Hiromasa Y, Wheeler S, Oppert C, Marshall SA, Tomich JM, Oppert

739 B, others. 2009. Identification, RNAi knockdown, and functional analysis of an ejaculate

$740 \quad$ protein that mediates a postmating, prezygotic phenotype in a cricket. PloS one 4:e7537-

$741 \quad$ e7546.

742 Marshall JL, Huestis DL, Garcia C, Hiromasa Y, Wheeler S, Noh S, Tomich JM, Howard DJ.

743 2011. Comparative proteomics uncovers the signature of natural selection acting on the

744 ejaculate proteomes of two cricket species isolated by postmating, prezygotic phenotypes.

$745 \quad$ Molecular biology and evolution 28:423-435.

746 Marshall JL, DiRienzo N. 2012. Noncompetitive gametic isolation between sibling species of a

747 cricket: a hypothesized link between within-population incompatibility and reproductive

748 isolation between species. International Journal of Evolutionary Biology 2012:593438.

749 McCartney MA, Lessios HA. 2004. Adaptive evolution of sperm bindin tracks egg

750 incompatibility in neotropical sea urchins of the genus Echinometra. Molecular Biology

$751 \quad$ and Evolution 21:732-745.

752 McDonald JH, Kreitman M, others. 1991. Adaptive protein evolution at the Adh locus in

753 Drosophila. Nature 351:652-654.

754 Metta M, Gudavalli R, Gibert J-M, Schlötterer C. 2006. No accelerated rate of protein evolution

755 in male-biased Drosophila pseudoobscura genes. Genetics 174:411-420.

756 Mueller JL, Ripoll DR, Aquadro CF, Wolfner MF. 2004. Comparative structural modeling and

757 inference of conserved protein classes in Drosophila seminal fluid. Proceedings of the

$758 \quad$ National Academy of Sciences 101:13542-13547. 
759 Mueller JL, Ram KR, McGraw LA, Qazi MB, Siggia ED, Clark AG, Aquadro CF, Wolfner MF.

760 2005. Cross-species comparison of Drosophila male accessory gland protein genes.

$761 \quad$ Genetics 171:131-143.

762 Murrell B, Weaver S, Smith MD, Wertheim JO, Murrell S, Aylward A, Eren K, Pollner T,

763 Martin DP, Smith DM, Scheffler K, Kosakovsky Pond, Sergei L. SLK. 2015. Gene-wide

764 identification of episodic selection. Molecular Biology and Evolution 32:1365-1371.

765 Muse SV, Gaut BS. 1994. A likelihood approach for comparing synonymous and

766 nonsynonymous nucleotide substitution rates, with application to the chloroplast genome.

767 Molecular Biology and Evolution 11:715-724.

768 Neilson LI, Schneider PA, Van Deerlin PG, Kiriakidou M, Driscoll DA, Pellegrini MC,

769 Millinder S, Yamamoto KK, French CK, Strauss JF. 1999. cDNA cloning and

770 characterization of a human sperm antigen (SPAG6) with homology to the product of the

771 Chlamydomonas PF16 locus. Genomics 60:272-280.

772 Nielsen R. 2001. Statistical tests of selective neutrality in the age of genomics. Heredity 86:641-

$773 \quad 647$.

774 Nielsen R. 2005. Molecular signatures of natural selection. Annual Review of Genetics 39:197-

218.

776 Niksirat H, James P, Andersson L, Kouba A, Kozák P. 2015. Label-free protein quantification in

777 freshly ejaculated versus post-mating spermatophores of the noble crayfish Astacus

778 astacus. Journal of Proteomics 123:70-77.

779 Noguchi M, Sawada T, Akazawa T. 2001. ATP-regenerating system in the cilia of Paramecium

780 caudatum. Journal of Experimental Biology 204:1063-1071. 
781 Noor MAF, Feder JL. 2006. Speciation genetics: evolving approaches. Nature Reviews Genetics 782 $7: 851-861$.

783 Nosil P, Schluter D. 2011. The genes underlying the process of speciation. Trends in Ecology \& 784 Evolution 26:160-167.

785 Osanai M, Chen PS. 1993. A comparative study on the arginine degradation cascade for sperm maturation of Bombyx mori and Drosophila melanogaster. Amino Acids 5:341-350.

787

788

789

790

791

792

793

794

795

796

797

798

799

800

801

802

Pamilo P, Nei M. 1988. Relationships between gene trees and species trees. Molecular Biology and Evolution 5:568-583.

Panhuis TM, Swanson WJ. 2006. Molecular evolution and population genetic analysis of candidate female reproductive genes in Drosophila. Genetics 173:2039-2047.

Payseur BA. 2010. Using differential introgression in hybrid zones to identify genomic regions involved in speciation. Molecular Ecology Resources 10:806-820.

Poiani A. 2006. Complexity of seminal fluid: a review. Behavioral Ecology and Sociobiology 60:289-310.

Poland V, Eubel H, King M, Solheim C, Harvey Millar A, Baer B. 2011. Stored sperm differs from ejaculated sperm by proteome alterations associated with energy metabolism in the honeybee Apis mellifera. Molecular Ecology 20:2643-2654.

Prokupek A, Hoffmann F, Eyun S, Moriyama E, Zhou M, Harshman L. 2008. An evolutionary expressed sequence tag analysis of Drosophila spermatheca genes. Evolution 62:29362947.

Pröschel M, Zhang Z, Parsch J. 2006. Widespread adaptive evolution of Drosophila genes with sex-biased expression. Genetics 174:893-900. 
803 Pruett PS, Azzi A, Clark SA, Yousef MS, Gattis JL, Somasundaram T, Ellington WR, Chapman

804 MS. 2003. The putative catalytic bases have, at most, an accessory role in the mechanism 805 of arginine kinase. Journal of Biological Chemistry 278:26952-26957.

806 Ram KR, Ji S, Wolfner MF. 2005. Fates and targets of male accessory gland proteins in mated 807 female Drosophila melanogaster. Insect Biochemistry and Molecular Biology 35:1059$808 \quad 1071$.

809 Ramm SA, Oliver PL, Ponting CP, Stockley P, Emes RD. 2008. Sexual selection and the 810 adaptive evolution of mammalian ejaculate proteins. Molecular Biology and Evolution $811 \quad 25: 207-219$.

812 Ram KR, Wolfner MF. 2007. Seminal influences: Drosophila Acps and the molecular interplay 813 between males and females during reproduction. Integrative and Comparative Biology 814 $47: 427-445$.

815 Rieseberg LH, Church SA, Morjan CL. 2004. Integration of populations and differentiation of species. New Phytologist 161:59-69.

817 Sapiro R, Kostetskii I, Olds-Clarke P, Gerton GL, Radice GL, III JFS. 2002. Male infertility, 818 impaired sperm motility, and hydrocephalus in mice deficient in Sperm-Associated 819 Antigen 6. Molecular and Cellular Biology 22:6298-6305.

820 Schnakenberg SL, Matias WR, Siegal ML. 2011. Sperm-storage defects and live birth in $821 \quad$ Drosophila females lacking spermathecal secretory cells. PLoS Biology 9:e1001192.

822 Schnakenberg SL, Siegal ML, Bloch Qazi MC. 2012. Oh, the places they'll go: female sperm 823 storage and sperm precedence in Drosophila melanogaster. Spermatogenesis 2:224-235. 
824 Shriner D, Nickle DC, Jensen MA, Mullins JI. 2003. Potential impact of recombination on

825 sitewise approaches for detecting positive natural selection. Genetical Research 81:115-

$826 \quad 121$.

827 Snook RR, Chapman T, Moore PJ, Wedell N, Crudgington HS. 2009. Interactions between the

828 sexes: new perspectives on sexual selection and reproductive isolation. Evolutionary

$829 \quad$ Ecology 23:71-91.

830 Springer SA, Crespi BJ. 2007. Adaptive gamete-recognition divergence in a hybridizing Mytilus

$831 \quad$ population. Evolution 61:772-783.

832 Strong SJ, Ellington WR. 1993. Horseshoe crab sperm contain a unique isoform of arginine

833 kinase that is present in the midpiece and flagellum. Journal of Experimental Zoology

$834 \quad 267: 563-571$.

835 Sun J, Spradling AC. 2013. Ovulation in Drosophila is controlled by secretory cells of the

836 female reproductive tract. eLife 2:e00415.

837 Swanson WJ, Clark AG, Waldrip-Dail HM, Wolfner MF, Aquadro CF. 2001. Evolutionary EST

838 analysis identifies rapidly evolving male reproductive proteins in Drosophila.

$839 \quad$ Proceedings of the National Academy of Sciences 98:7375-7379.

840 Swanson WJ, Wong A, Wolfner MF, Aquadro CF. 2004. Evolutionary expressed sequence tag

$841 \quad$ analysis of Drosophila female reproductive tracts identifies genes subjected to positive

$842 \quad$ selection. Genetics 168:1457-1465.

843 Swanson WJ, Vacquier VD. 2002. The rapid evolution of reproductive proteins. Nature Reviews

$844 \quad$ Genetics 3:137-144.

845 Tamura K, Stecher G, Peterson D, Filipski A, Kumar S. 2013. MEGA6: Molecular Evolutionary

846 Genetics Analysis version 6.0. Molecular Biology and Evolution 30:2725-2729. 
847 Tamura K, Nei M. 1993. Estimation of the number of nucleotide substitutions in the control

848 region of the mitochondrial DNA in humans and chimpanzees. Molecular Biology and $849 \quad$ Evolution 10:512-526.

850 Templeton AR, Crandall KA, Sing CF. 1992. A cladistic analysis of phenotypic associations 851 with haplotypes inferred from restriction endonuclease mapping and DNA sequence data.

$852 \quad$ III. Cladogram estimation. Genetics 132:619-633.

853 Ting C-T, Tsaur S-C, Wu C-I. 2000. The phylogeny of closely related species as revealed by the 854 genealogy of a speciation gene, Odysseus. Proceedings of the National Academy of $855 \quad$ Sciences 97:5313-5316.

856 Traylor T, Birand AC, Marshall JL, Howard DJ. 2008. A zone of overlap and hybridization 857 between Allonemobius socius and a new Allonemobius sp. Annals of the Entomological $858 \quad$ Society of America 101:30-39.

859 Turner LM, Chuong EB, Hoekstra HE. 2008. Comparative analysis of testis protein evolution in $860 \quad$ rodents. Genetics 179:2075-2089.

861 Uda K, Fujimoto N, Akiyama Y, Mizuta K, Tanaka K, Ellington WR, Suzuki T. 2006. Evolution 862 of the arginine kinase gene family. Comparative Biochemistry and Physiology Part D: $863 \quad$ Genomics and Proteomics 1:209-218.

864 Visconti PE, Krapf D, de la Vega-Beltrán JL, Acevedo JJ, Darszon A. 2011. Ion channels, 865 phosphorylation and mammalian sperm capacitation. Asian Journal of Andrology

866 $13: 395-405$.

867 Wagstaff BJ, Begun DJ. 2005. Molecular population genetics of accessory gland protein genes 868 and testis-expressed genes in Drosophila mojavensis and D. arizonae. Genetics 171:1083-1101. 
870 Walters JR, Harrison RG. 2010. Combined EST and proteomic analysis identifies rapidly

871 evolving seminal fluid proteins in Heliconius butterflies. Molecular biology and

872 evolution 27:2000-2013.

873 Wang R-L, Hey J. 1996. The speciation history of Drosophila pseudoobscura and close relatives:

874 inferences from DNA sequence variation at the period locus. Genetics 144:1113-1126.

875 Wolfner MF. 2011. Precious essences: female secretions promote sperm storage in Drosophila. $876 \quad$ PLoS Biology 9:e1001191.

877 Wu C-I. 2001. The genic view of the process of speciation. Journal of Evolutionary Biology $878 \quad 14: 851-865$.

879 Wu C-I, Ting C-T. 2004. Genes and speciation. Nature Reviews Genetics 5:114-122.

880 Yang Z, Bielawski JP. 2000. Statistical methods for detecting molecular adaptation. Trends in $881 \quad$ Ecology \& Evolution 15:496-503.

882 Zhang Z, Hambuch TM, Parsch J. 2004. Molecular evolution of sex-biased genes in Drosophila. 883 Molecular Biology and Evolution 21:2130-2139.

884 Zhang Z, Parsch J. 2005. Positive correlation between evolutionary rate and recombination rate 885 in Drosophila genes with male-biased expression. Molecular Biology and Evolution $886 \quad 22: 1945-1947$.

887 Zhou G, Somasundaram T, Blanc E, Parthasarathy G, Ellington WR, Chapman MS. 1998.

888 Transition state structure of arginine kinase: implications for catalysis of bimolecular 889 reactions. Proceedings of the National Academy of Sciences 95:8449-8454.

890 Zmasek CM, Eddy SR. 2011. A simple algorithm to infer gene duplication and speciation events $891 \quad$ on a gene tree. Bioinformatics 17:821-828.

892 


\section{Table $\mathbf{1}$ (on next page)}

Nucleotide variation within each $A$. socius complex species

Table 1. Nucleotide variation within each $A$. socius complex species. A total of 3 populations of $A$. fasciatus, 7 populations of $A$. socius, and 3 populations of $A$. sp. nov. Tex were sampled. The number of individuals sampled for each gene are shown with the number of populations they came from ( $n(p o p)) .\left(\pi_{s}\right.$ : the average number of synonymous nucleotide differences per site for any random pair of sequences; $\pi_{a}$ : the average number of non-synonymous nucleotide differences per site for any random pair of sequences; $\theta$ : a metric of the population substitution rate; all metrics were calculated across populations) 
1 Table 1. Nucleotide variation within each $A$. socius complex species. A total of 3 populations of $A$. fasciatus, 7 populations of $A$.

2 socius, and 3 populations of $A$. sp. nov. Tex were sampled. The number of individuals sampled for each gene are shown with the

3 number of populations they came from (n (pop)). ( $\pi_{\mathrm{s}}$ : the average number of synonymous nucleotide differences per site for any

4 random pair of sequences; $\pi_{\mathrm{a}}$ : the average number of non-synonymous nucleotide differences per site for any random pair of

5 sequences; $\theta$ : a metric of the population substitution rate; all metrics were calculated across populations)

\begin{tabular}{|c|c|c|c|c|c|c|c|c|c|c|c|c|c|}
\hline \multirow[b]{2}{*}{ Gene } & \multirow[b]{2}{*}{ Length } & \multicolumn{4}{|c|}{ A. fasciatus } & \multicolumn{4}{|c|}{ A. socius } & \multicolumn{4}{|c|}{ A. sp. nov. Tex } \\
\hline & & $\mathrm{n}(\mathrm{pop})$ & $\pi_{\mathrm{s}}$ & $\pi_{\mathrm{a}}$ & $\theta_{\text {fas }}$ & $\mathrm{n}(\mathrm{pop})$ & $\pi_{\mathrm{s}}$ & $\pi_{\mathrm{a}}$ & $\theta_{\text {soc }}$ & $\mathrm{n}$ (pop) & $\pi_{\mathrm{s}}$ & $\pi_{\mathrm{a}}$ & $\theta_{\mathrm{Tex}}$ \\
\hline ACG69 & 414 & $9(3)$ & 0.005 & 0.004 & 0.007 & $14(6)$ & 0.021 & 0.009 & 0.011 & $7(3)$ & 0 & 0 & 0 \\
\hline $\mathrm{AK}$ & 1173 & $9(3)$ & 0.002 & $<0.001$ & 0.001 & $15(6)$ & 0.004 & 0.001 & 0.002 & $6(3)$ & 0.003 & 0.001 & 0.002 \\
\hline APBP & 705 & $9(3)$ & 0.001 & 0 & 0.001 & $15(5)$ & 0.005 & 0 & 0.001 & $8(3)$ & 0 & 0 & $\overline{0}$ \\
\hline EJAC- & 726 & $9(3)$ & 0 & 0 & 0 & $16(6)$ & 0.001 & $<0.001$ & 0.001 & $9(3)$ & 0.003 & 0 & 0.001 \\
\hline \multicolumn{14}{|l|}{ SP } \\
\hline GOT & 1122 & $9(3)$ & 0.002 & 0 & $<0.001$ & $17(7)$ & 0 & 0 & 0 & $9(3)$ & 0.005 & 0.001 & 0.002 \\
\hline SPAG6 & 426 & $9(3)$ & 0 & 0 & 0 & $17(6)$ & 0 & 0 & 0 & $8(3)$ & 0.005 & 0 & 0.001 \\
\hline SPI & 315 & $9(3)$ & 0.007 & 0 & 0.002 & $16(6)$ & 0 & 0 & 0 & $9(3)$ & 0.008 & 0.001 & 0.002 \\
\hline
\end{tabular}

6 


\section{Table 2 (on next page)}

Nucelotide variation at each branching node of the $A$. socius complex species tree.

Table 2. Nucelotide variation at each branching node of the $A$. socius complex species tree. $\left(P_{\mathrm{N}}\right.$ : non-synonymous polymorphisms; $P_{\mathrm{S}}$ : synonymous polymorphisms; $D_{\mathrm{N}}$ : non-synonymous fixations; $D_{s}$ : synonymous fixations; $\kappa_{s}$ : rate of non-synonymous substitutions per nonsynonymous site; $k_{a}$ : rate of synonymous substitutions per synonymous site; $\omega=k_{a} / k_{s}$ : estimate from maximum likelihood model fit in HyPhy, along with 95\% asymptotic normal confidence intervals $(\mathrm{Cl})$ ) 
1 Table 2. Nucelotide variation at each branching node of the $A$. socius complex species tree. $\left(P_{\mathrm{N}}\right.$ : non-synonymous polymorphisms; $P_{\mathrm{S}}$ :

2 synonymous polymorphisms; $D_{\mathrm{N}}$ : non-synonymous fixations; $D_{\mathrm{S}}$ : synonymous fixations; $\kappa_{\mathrm{s}}$ : rate of non-synonymous substitutions per

3 non-synonymous site; $\kappa_{\mathrm{a}}$ : rate of synonymous substitutions per synonymous site; $\omega=\kappa_{\mathrm{a}} / \kappa_{\mathrm{s}}$ : estimate from maximum likelihood model

4 fit in HyPhy, along with 95\% asymptotic normal confidence intervals (CI))

\begin{tabular}{|c|c|c|c|c|c|c|c|c|c|c|c|c|c|c|c|}
\hline \multirow[b]{2}{*}{ Gene } & \multirow[b]{2}{*}{ Length } & \multicolumn{7}{|c|}{ between $A$. fasciatus \& (A. socius $+A$. sp. nov. Tex $)$} & \multicolumn{7}{|c|}{ between $A$. socius \& A. sp. nov. Tex } \\
\hline & & $P_{\mathrm{N}}$ & $P_{\mathrm{S}}$ & $D_{\mathrm{N}}$ & $D_{\mathrm{S}}$ & $\kappa_{\mathrm{s}}$ & $\kappa_{\mathrm{a}}$ & $\omega(95 \% \mathrm{CI})$ & $P_{\mathrm{N}}$ & $P_{\mathrm{S}}$ & $D_{\mathrm{N}}$ & $D_{\mathrm{S}}$ & $\kappa_{\mathrm{s}}$ & $\kappa_{\mathrm{a}}$ & $\omega(95 \% \mathrm{CI})$ \\
\hline ACG69 & 414 & 7 & 6 & 0 & 0 & 0.024 & 0.009 & $0.572(0,1.366)$ & 7 & 6 & 0 & 0 & 0.021 & 0.007 & $0.142(0,0.420)$ \\
\hline $\mathrm{AK}$ & 1173 & 3 & 12 & 2 & 0 & 0.006 & 0.003 & $24.841(12.988,36.694$ & 2 & 9 & 1 & 2 & 0.011 & 0.002 & $0.147(0,0.436)$ \\
\hline APBP & 705 & 1 & 4 & 1 & 0 & 0.005 & 0.003 & $13.495(0,35.670)$ & 0 & 3 & 1 & 0 & 0.007 & 0.002 & $0.285(0,0.843)$ \\
\hline EJAC-SP & 726 & 1 & 3 & 0 & 0 & 0.008 & 0.001 & $0.993(0,16.673)$ & 1 & 2 & 0 & 1 & 0.014 & 0.002 & $0.158(0,0.467)$ \\
\hline GOT & 1122 & 3 & 7 & 1 & 1 & 0.011 & 0.002 & $0.274(0,0.811)$ & 3 & 4 & 0 & 2 & 0.011 & 0.001 & $0.136(0,0.402)$ \\
\hline SPAG6 & 426 & 0 & 1 & 0 & 2 & 0.029 & 0 & $0(0,0.001)$ & 0 & 1 & 0 & 0 & 0.004 & 0 & $0.001(0.001,0.002)$ \\
\hline SPI & 315 & 3 & 4 & 0 & 0 & 0.02 & 0.003 & $1.356(0$, inf $)$ & 1 & 1 & 2 & 3 & 0.054 & 0.009 & $0.178(0,0.425)$ \\
\hline
\end{tabular}

5

6 


\section{Table 3(on next page)}

Genealogical sorting index values based on individual rooted gene trees

Table 3. Genealogical sorting index values based on individual rooted gene trees. Values range from zero (complete polyphyly) to one (complete monophyly). P-values are obtained from 10,000 permutations that randomly reassign tip labels on the tree and represent the probability of observing a tree with a gsi value more extreme than the current toplogy. 
1 Table 3. Genealogical sorting index values based on individual rooted gene trees. Values range

2 from zero (complete polyphyly) to one (complete monophyly). P-values are obtained from

310,000 permutations that randomly reassign tip labels on the tree and represent the probability of

4 observing a tree with a gsi value more extreme than the current toplogy.

\begin{tabular}{|c|c|c|c|c|c|c|}
\hline Gene & gsi-fas & $\mathrm{P}_{\text {perm }}$ & gsi-soc & $\mathrm{P}_{\text {perm }}$ & gsi-Tex & $\mathrm{P}_{\text {perm }}$ \\
\hline ACG69 & 0.458 & $<0.001$ & 0 & 1 & 0.836 & $<0.001$ \\
\hline$\overline{\mathrm{AK}}$ & 1 & $<0.001$ & 0.843 & $<0.001$ & 1 & $<0.001$ \\
\hline APBP & 1 & $<0.001$ & 0.849 & $<0.001$ & 1 & $<0.001$ \\
\hline EJAC-SP & 0.609 & $<0.001$ & 0.730 & $<0.001$ & 0.288 & 0.001 \\
\hline GOT & 0.844 & $<0.001$ & 0.533 & $<0.001$ & 1 & $<0.001$ \\
\hline SPAG6 & 1 & $<0.001$ & 0.667 & $<0.001$ & 0.191 & 0.016 \\
\hline SPI & 0.189 & 0.010 & 0.622 & $<0.001$ & 1 & $<0.001$ \\
\hline
\end{tabular}

5

6 


\section{Table 4 (on next page)}

Summary of results found testing for evidence of selection in ejaculate protein coding genes in the $A$. socius complex

Table 4. Summary of results found testing for evidence of selection in ejaculate protein

coding genes in the $A$. socius complex. The specific method used for each result is shown in parentheses. 
1 Table 4. Summary of results found testing for evidence of selection in ejaculate protein coding genes in the $A$. socius complex. The

2 specific method used for each result is shown in parentheses.

\begin{tabular}{|c|c|c|c|c|c|c|c|c|}
\hline Genes & $\begin{array}{l}\text { Species } \\
\text { tree } \pi_{\mathrm{s}}, \pi_{\mathrm{a}}, \theta\end{array}$ & $\begin{array}{l}\text { Species } \\
\text { tree } \omega \\
(\mathrm{MLE})\end{array}$ & $\begin{array}{l}D_{\mathrm{N}} / D_{\mathrm{S}}> \\
P_{\mathrm{N}} / P_{\mathrm{S}}(\mathrm{MK})\end{array}$ & $\begin{array}{l}\text { Departure } \\
\text { from other } \\
\text { genes } \\
\text { (HKA) }\end{array}$ & $\begin{array}{l}\text { Gene tree } \\
\text { evidence of } \\
\text { branch } \\
\text { selection } \\
\text { (TestBranc } \\
\text { hdNdS) }\end{array}$ & $\begin{array}{l}\text { Gene tree } \\
\text { evidence of } \\
\text { site } \\
\text { selection } \\
\text { (SLAC) }\end{array}$ & $\begin{array}{l}\text { Lineage } \\
\text { sorting } \\
\text { (allopatric } \\
+ \text { contact } \\
\text { zone) (gsi) }\end{array}$ & $\begin{array}{l}\text { Allelic } \\
\text { segregation } \\
\text { in contact } \\
\text { zone }(D)\end{array}$ \\
\hline $\begin{array}{l}\text { ACG6 } \\
9\end{array}$ & small & all $<1$ & $\mathrm{NA}$ & no & no & no & low & low \\
\hline $\mathrm{AK}$ & smaller & some $>1$ & no & no & yes & no & high & high \\
\hline APBP & smaller & some $>1$ & no & no & yes & no & high & high \\
\hline $\begin{array}{l}\text { EJAC- } \\
\text { SP }\end{array}$ & smaller & some $\sim 1$ & NA & no & no & $\begin{array}{l}\text { purifying } \\
\text { selection }\end{array}$ & low & high \\
\hline GOT & smaller & all $<1$ & no & maybe & no & $\begin{array}{l}\text { purifying } \\
\text { selection }\end{array}$ & moderate & low \\
\hline $\begin{array}{l}\text { SPAG } \\
6\end{array}$ & smaller & all $<1$ & NA & no & no & no & low & high \\
\hline SPI & smaller & some $>1$ & no & no & no & no & low & low \\
\hline
\end{tabular}

3 


\section{1}

Neighbor-Joining gene trees that include allopatric individuals only.

Figure 1. Neighbor-Joining gene trees that include allopatric individuals only. Internal branches whose non-synonymous substitution rate confidence intervals did not overlap with zero are marked. These internal branches were tested for evidence of elevated rates of nonsynonymous substitutions (TestBranchdNdS) and for episodic selection among sites and branches (BUSTED). 

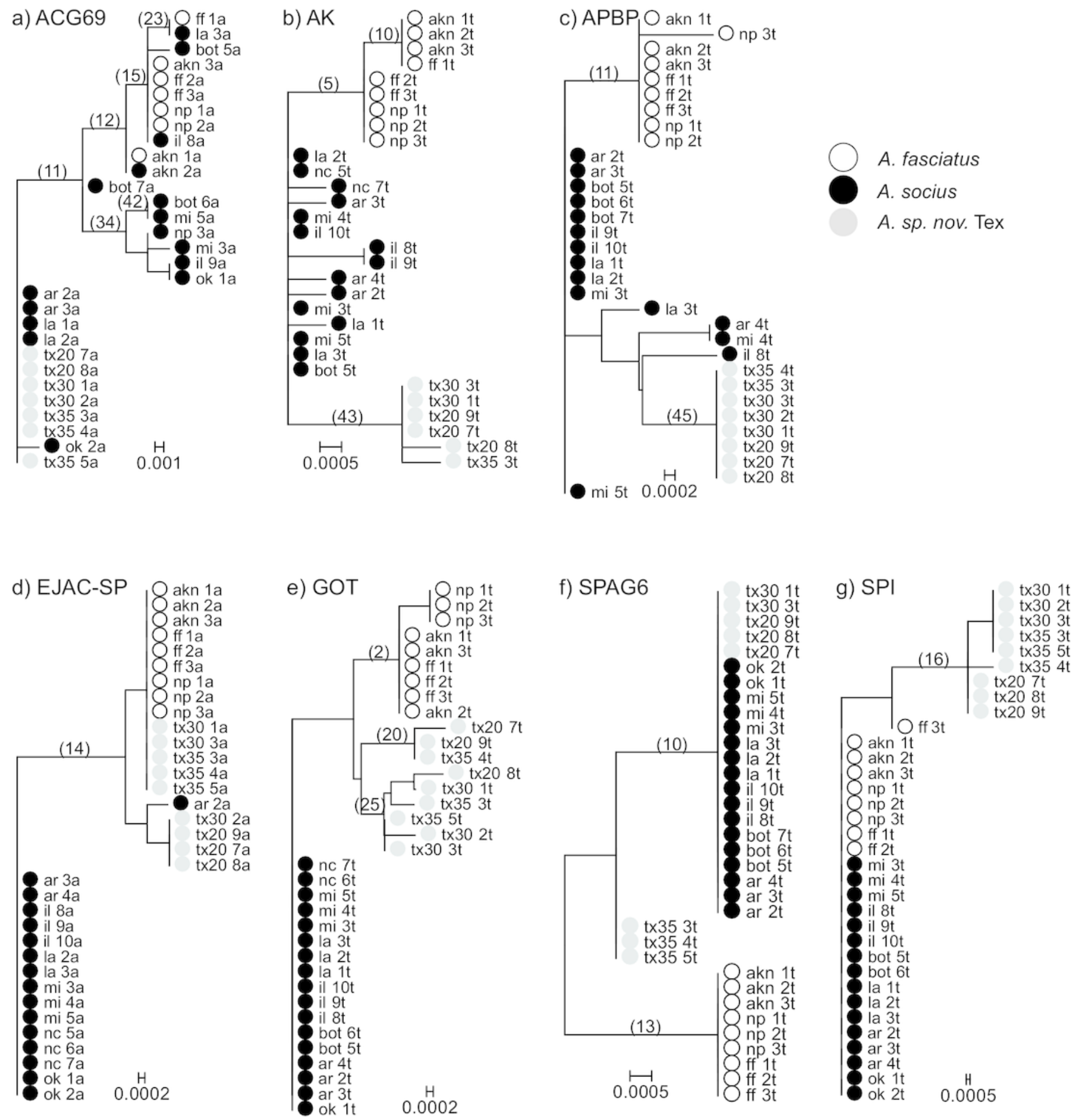


\section{Figure 2 (on next page)}

Rooted Neighbor-Joining gene trees that include both allopatric and contact zone individuals

Figure 2. Rooted Neighbor-Joining gene trees that include both allopatric and contact zone individuals. These trees were used to estimate degrees of lineage sorting for each species. 


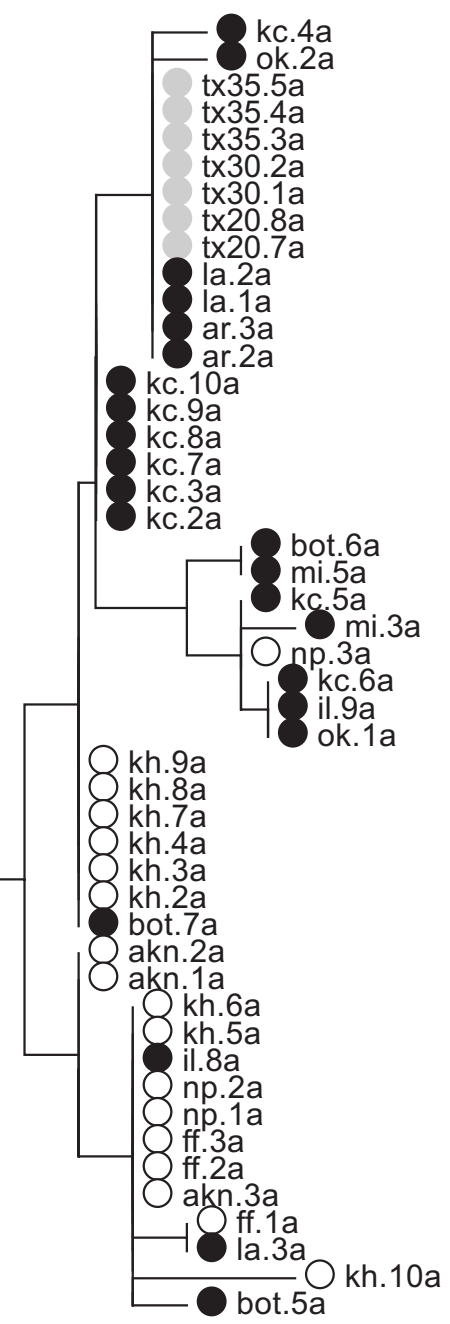

$\stackrel{\mapsto}{0.001}$

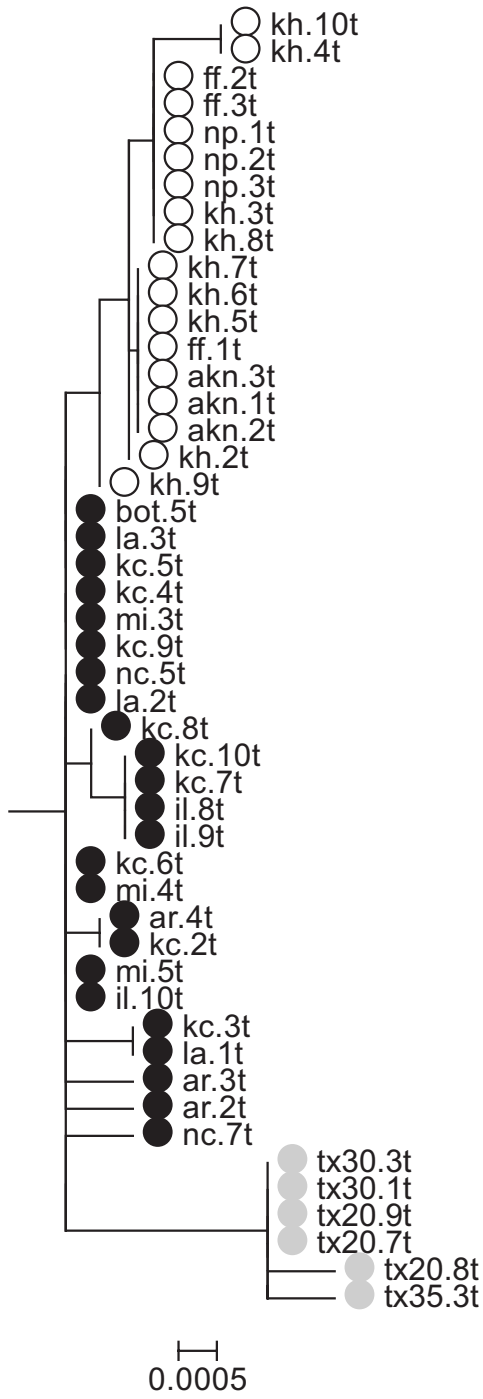

Peer) reviewing PDF | (2015:09:6569:1:1:NEW 9 Dec 2015)

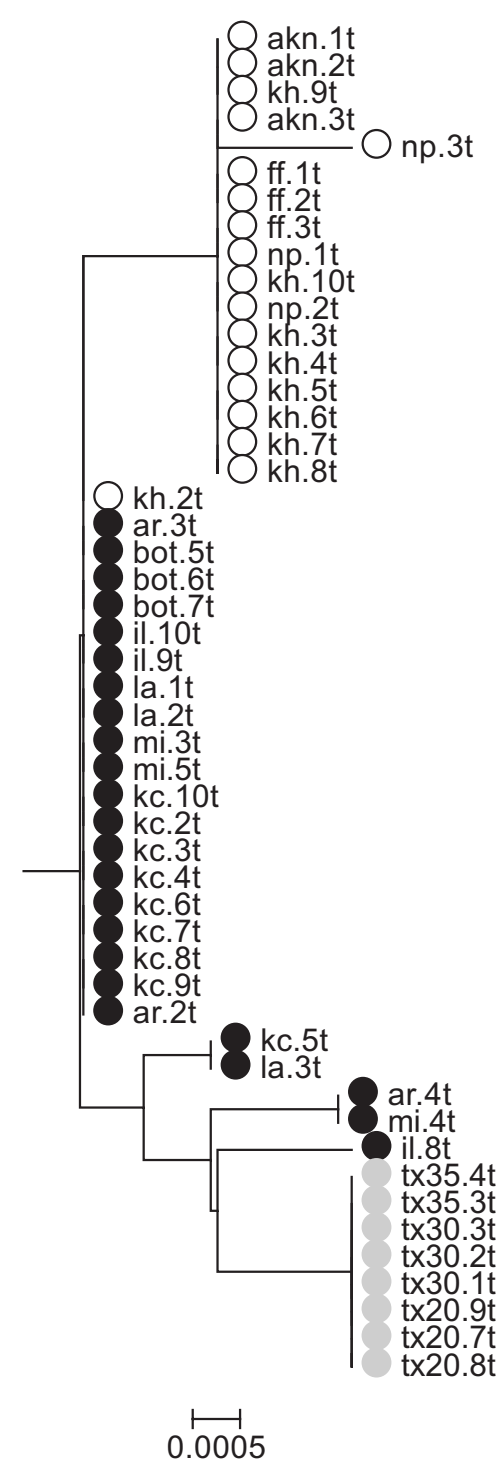




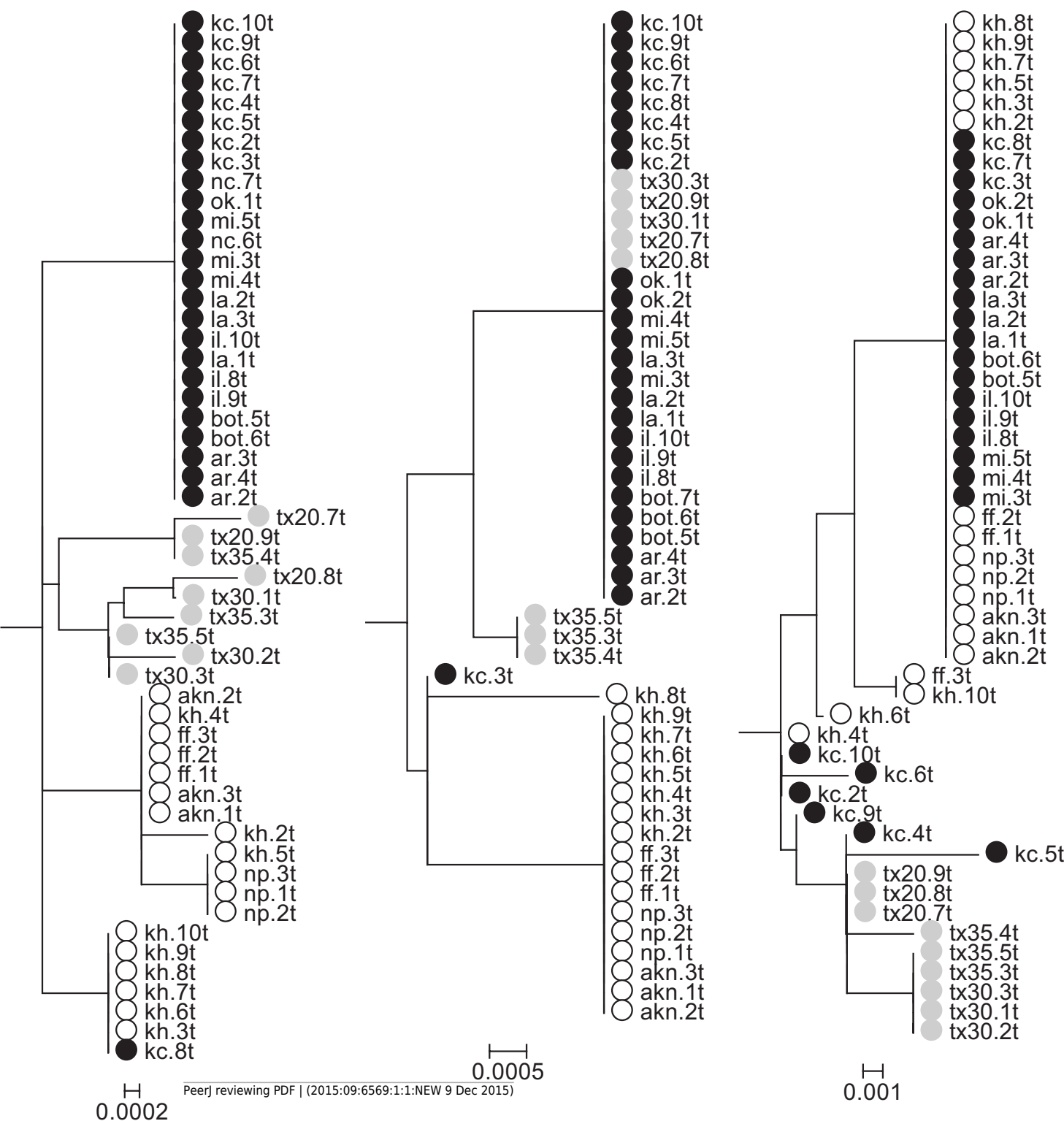


3

Statistical parsimony haplotype networks including only allopatric individuals

Figure 3. Statistical parsimony haplotype networks including only allopatric individuals, with mutational steps and nonsynonymous substitutions indicated. Population abbreviations are as in the main text. Sizes of each haplotype correspond to the relative number of individuals that possessed each haplotype. 
a) ACG69

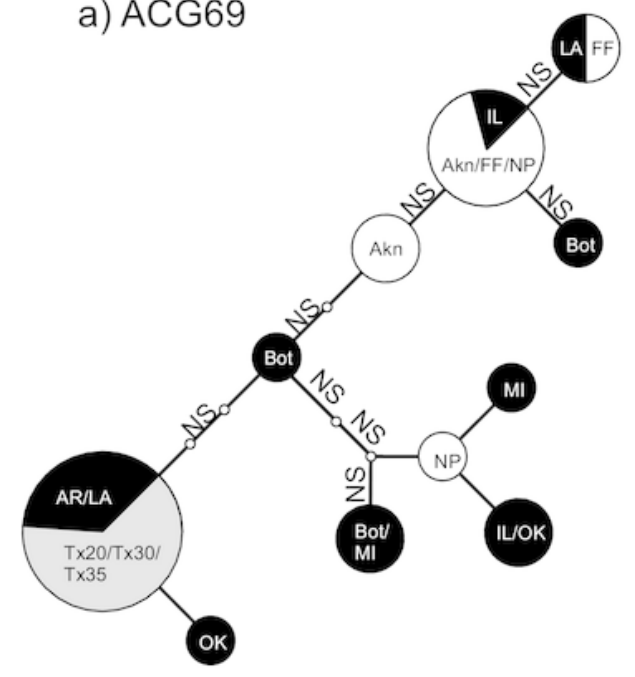

c) APBP

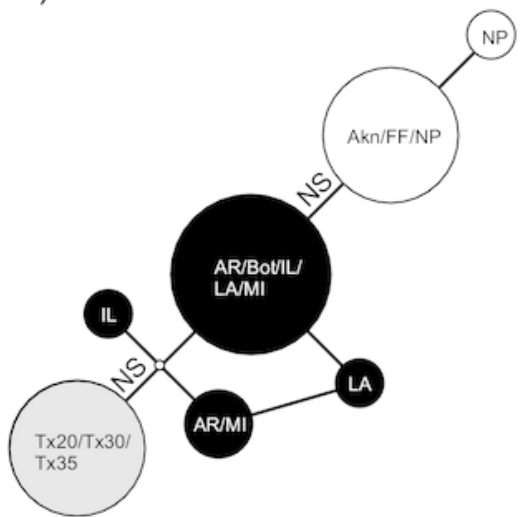

e) GOT

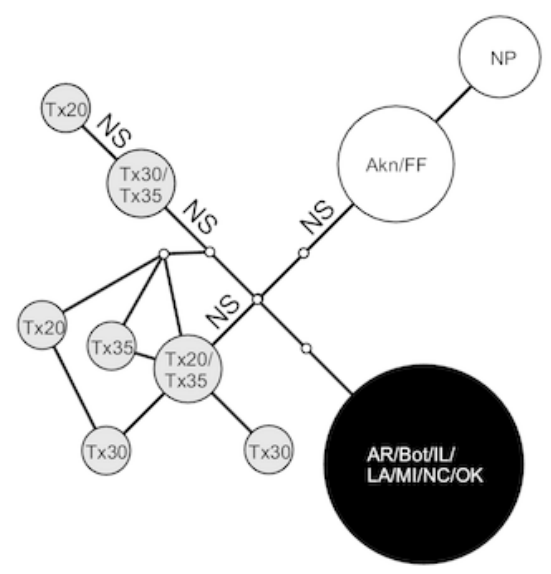

b) AK

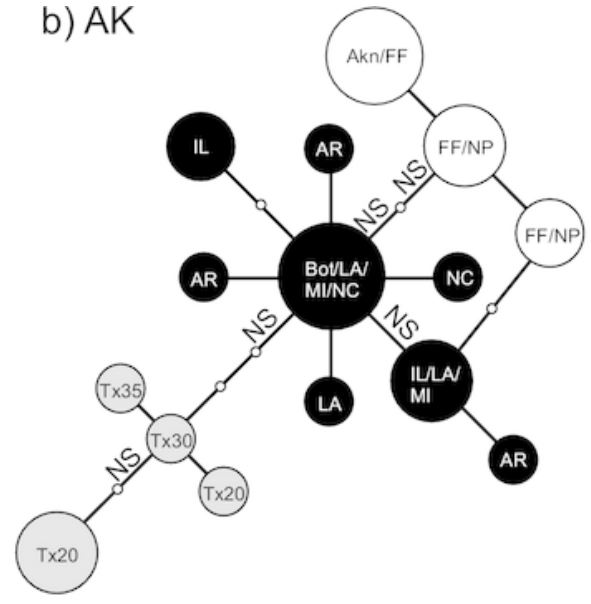

d) EJAC-SP

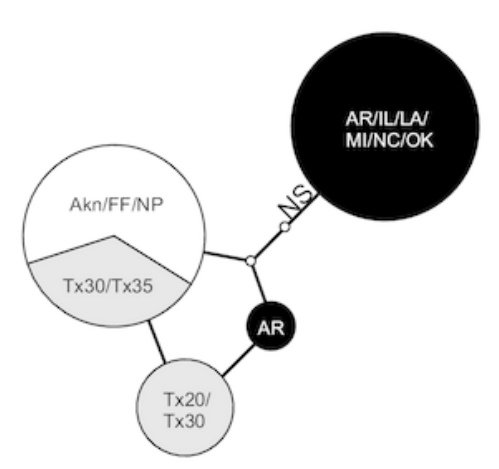

A. fasciatus

- A. socius

A. sp. nov. Tex

- assumed haplotype

NS nonsynonymous mutation

- 1 mutational step

f) SPAG6

g) SPI

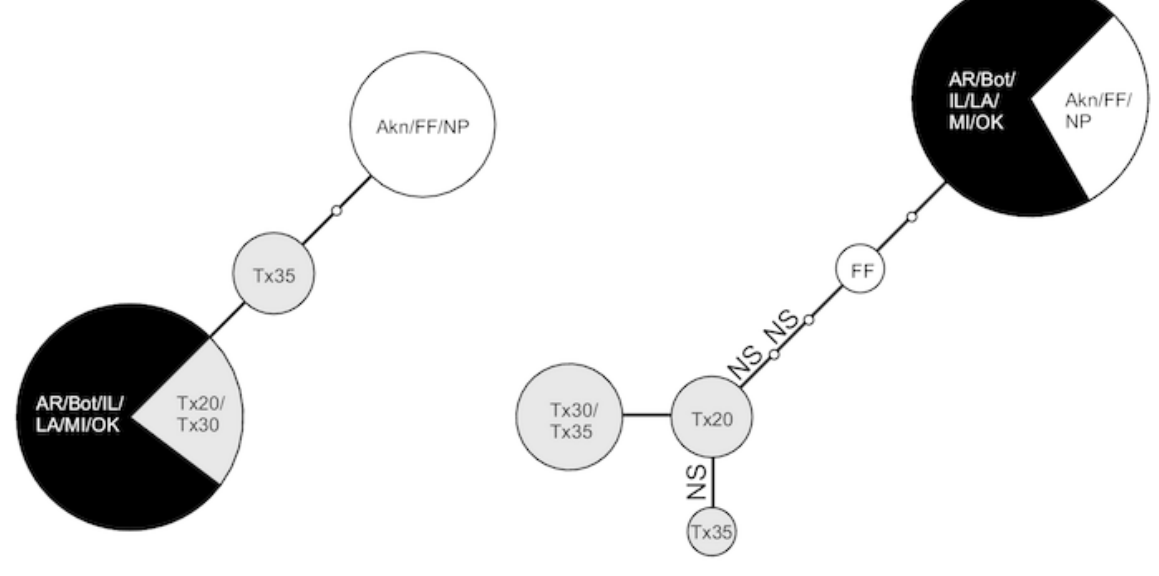


4

Distribution of species-specific vs. common (shared) alleles within the $A$. fasciatus and A. socius contact zone in Kenna, WV

Figure 4. Distribution of species-specific vs. common (shared) alleles within the A. fasciatus and $A$. socius contact zone in Kenna, WV. Nine individuals each with allozyme identities of pure (homozygous) $A$. fasciatus and $A$. socius had varying patterns of allelic identities for the seven genes. Numbers (2-9) indicate the sampled individual and letters ( $a \& b$ ) indicate the alleles within each individual. The dissimilarity index $D$ is a metric of the degree of allelic segregation between the two contact zone populations.

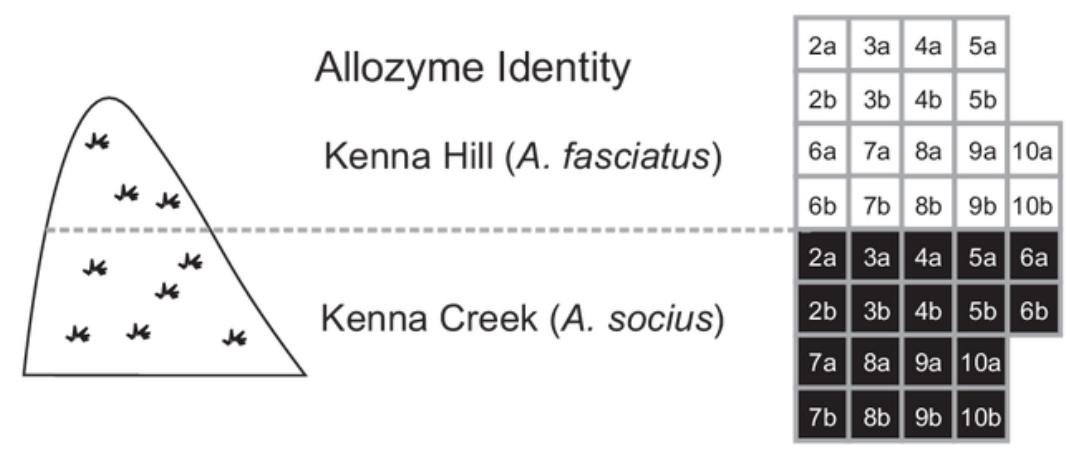

Allelic Identity

$\square$ fas allele

soc allele

common allele
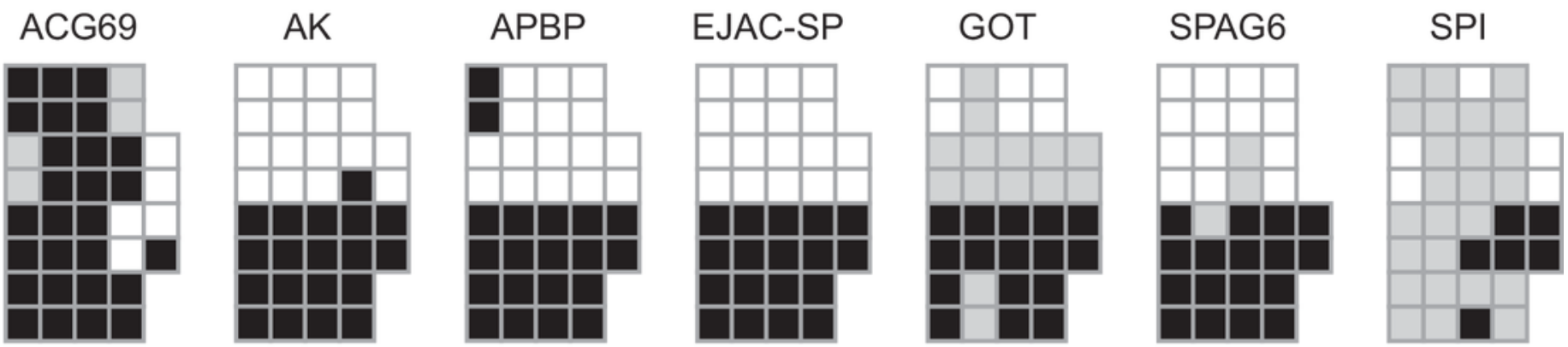

$D=0.164$

$D=0.944$

$D=0.889$

$D=1$

$D=0.353$

$D=0.771 \sim 0.886$

$D=0.317$ 